\title{
Mutations in EPHB4 cause human venous valve aplasia
}

Oliver Lyons, ${ }^{1}$ James Walker, ${ }^{1}$ Christopher Seet, ${ }^{1}$ Mohammed Ikram, ${ }^{1}$ Adam Kuchta, ${ }^{2}$ Andrew Arnold, ${ }^{2}$ Magda Hernández-Vásquez, ${ }^{3}$ Maike Frye, ${ }^{3}$ Gema Vizcay-Barrena, ${ }^{4}$ Roland A. Fleck, ${ }^{4}$ Ashish S. Patel, ${ }^{1}$ Soundrie Padayachee, ${ }^{2}$ Peter Mortimer, ${ }^{5}$ Steve Jeffery, ${ }^{5}$ Siren Berland, ${ }^{6}$ Sahar Mansour, ${ }^{5,7}$ Pia Ostergaard, ${ }^{5}$ Taija Makinen, ${ }^{3}$ Bijan Modarai, ${ }^{1}$ Prakash Saha, and Alberto Smith ${ }^{1}$

'Academic Department of Vascular Surgery, Section of Vascular Risk and Surgery, School of Cardiovascular Medicine and Sciences, BHF Centre of Research Excellence, King's College London, St. Thomas' Hospital, London, United Kingdom. ${ }^{2}$ Department of Ultrasonic Angiology, Guy's \& St. Thomas' NHS Foundation Trust, London, United Kingdom. ${ }^{3}$ Rudbeck Laboratory, Department of Immunology, Genetics and Pathology, Uppsala University, Sweden. ${ }^{4}$ Centre for Ultrastructural Imaging, King's College London, London, United Kingdom. ' Molecular and Clinical Sciences Research Institute, St. George's University of London, London, United Kingdom. ${ }^{6}$ Department of Medical Genetics, Haukeland University Hospital, Bergen, Norway. ${ }^{7}$ South West Thames Regional Genetics Service, St. George's Hospital, London, United Kingdom.

Venous valve (VV) failure causes chronic venous insufficiency, but the molecular regulation of valve development is poorly understood. A primary lymphatic anomaly, caused by mutations in the receptor tyrosine kinase EPHB4, was recently described, with these patients also presenting with venous insufficiency. Whether the venous anomalies are the result of an effect on VVs is not known. VV formation requires complex "organization" of valve-forming endothelial cells, including their reorientation perpendicular to the direction of blood flow. Using quantitative ultrasound, we identified substantial VV aplasia and deep venous reflux in patients with mutations in EPHB4. We used a GFP reporter in mice to study expression of its ligand, ephrinB2, and analyzed developmental phenotypes after conditional deletion of floxed Ephb4 and Efnb2 alleles. EphB4 and ephrinB2 expression patterns were dynamically regulated around organizing valve-forming cells. Efnb2 deletion disrupted the normal endothelial expression patterns of the gap junction proteins connexin37 and connexin43 (both required for normal valve development) around reorientating valve-forming cells and produced deficient valve-forming cell elongation, reorientation, polarity, and proliferation. Ephb4 was also required for valve-forming cell organization and subsequent growth of the valve leaflets. These results uncover a potentially novel cause of primary human $\mathrm{V}$ aplasia.

Conflict of interest: The authors have declared that no conflict of interest exists.

Copyright: (c) 2021, Lyons et al. This is an open access article published under the terms of the Creative Commons Attribution 4.0 International License.

Submitted: June 2, 2020

Accepted: August 11, 2021

Published: August 17, 2021

Reference information: /CI Insight. 2021;6(18):e140952.

https://doi.org/10.1172/jci.

insight.140952.

\section{Introduction}

Unidirectional blood flow requires functional venous valves (VVs), which are widely distributed throughout human veins and venules, predominantly in vessels less than $100 \mu \mathrm{m}$ in diameter (1). Lower limb VVs are typically bicuspid and situated just upstream of the confluence with a tributary $(1,2)$. Failure of these valves is the central feature of the venous reflux that is seen in up to $40 \%$ of adults $(3,4)$, although congenital VV aplasia has also been identified (5-8). In the lower limbs, venous reflux causes chronic venous hypertension, leading to pain, edema, hyperpigmentation, skin damage, and chronic intractable ulceration $(3,9,10)$. Our understanding of the molecular mechanisms of VV embryological development, maintenance after formation, and failure in disease is limited, and there are few therapeutic options to treat VV dysfunction (3, 11-16). Elucidating these mechanisms and understanding how their dysfunction may lead to VV failure could facilitate the development of novel therapies.

Clinical studies have suggested a link between venous reflux and some primary lymphedemas, and we have previously shown striking human VV disease in patients with primary lymphedema caused by mutations in FOXC2 (MIM 602402) and GJC2 (MIM 608803; refs. 11, 17-20). Other human genetics studies have shown that mutations in the gene encoding the tyrosine kinase receptor EPHB4 (EPHB4, MIM 618196) cause capillary malformation-arteriovenous malformation syndrome (CM-AVM2, including 
hereditary hemorrhagic telangiectasia and vein of Galen malformations, cutaneous malformations and arteriovenous malformations) and a primary lymphatic anomaly, which includes clinical features such as central conduction lymphatic anomaly, nonimmune fetal hydrops, and atrial septal defects (21-28). Patients with the primary lymphatic anomaly were also reported to present with varicose veins and early onset venous stasis $(21,25,28)$. In mice, early embryonic deletion of Ephb4 in lymphatic endothelia leads to subcutaneous edema and abnormal dermal and mesenteric lymphatic vasculature, whereas deletion in adult blood endothelia results in coronary abnormalities including capillary microhemorrhages $(21,29)$

The Eph receptors are the largest family of mammalian receptor tyrosine kinases and bind to ephrins, their transcellular ligands $(30,31)$. Cell-cell signaling may occur in either direction, resulting in cell and context-specific effects, and is involved in regulating many developmental processes including cell sorting and boundary formation (32-34). In the cardiovascular system, ephrinB2 is widely accepted as an arterial-specific marker, whereas EphB4 is used as a marker of venous endothelia (35-37). EphrinB2/EphB4 signaling is essential for developmental angiogenesis, and global knockout of Ephb4 is phenotypically similar to knockout of Efnb2, with both resulting in vascular remodeling defects and embryonic lethality $(35,38-40)$. Constitutive overexpression of ephrinB2 leads to defects including abnormal intersomitic vessel patterning, aortic dissection and aneurysm formation, and early neonatal lethality due to aortic rupture (41)

Signaling between ephrinB2 and EphB4 is required for lymphatic valve (LV) development and maintenance, and for formation of valves at lymphovenous junctions at the base of the neck $(12,21,42)$. LV cells fail to take on normal morphology in Efnb2 $2^{\Delta V / \Delta V}$ mice (lacking the C-terminal PDZ interaction site), and it was suggested that ephrinB2/EphB4 signaling is required to guide endothelial cell (EC) migration and elongation during LV morphogenesis (42). Blocking the forward signaling activity of EphB4 results in failure of LV formation $(43,44)$. Defects in cardiac valve (CV) development leading to early perinatal death are found in $E f n b 2^{\beta g a l / \beta g a l}$ mice, in which the cytoplasmic tail of ephrinB2 is replaced with $\beta$ gal (45). In both LV and CV, the morphological effects of loss (or inhibition) of ephrinB2/EphB4 signaling on Prox $1^{\text {hi }}$ valve-forming cells (VFCs) remain unclear. Ephrin-Eph interactions result in rapid changes in cellular direction and motility, leading to boundary formation within initially mixed populations of cells (for example, in mesenchymal cells), and can inhibit communication via gap junctions across these boundaries $(31,32,46)$. In vitro, ephrinB2/EphB4 signaling controls EC repulsion and segregation, leading to clustering of EphB4-expressing or ephrinB2-expressing cells, akin to in vivo boundary formation, but to the best of our knowledge this behavior has not been observed in ECs in vivo (47)

We previously showed that ephrinB2 is required for postnatal VV leaflet development and maintenance, but the expression of ephrinB2 and EphB4, and any roles in the early organization of VFCs, has not been examined $(11,12)$. In this study, we show that mutations in EPHB4 caused striking human VV disease, with an almost complete loss of VVs seen in some patients. Given the known roles for ephrinEph interactions in boundary formation in other tissues, we hypothesized that ephrin-Eph interactions could regulate early organizational events in VV formation. We have therefore focused on their respective roles in the regulation of the complex series of events during early valve formation in mice, which includes the organization of a set of Prox $1^{\text {hi }}$ VFCs to form a ring of cells within the 3D lumen of the vessel (stage 1 of development; refs. 11, 12). On postnatal day 0 (P0) this structure is found predominantly on the anterior vein wall and then extends posteriorly (11). Using a GFP reporter we identified Efnb2 expression within veins at the site of VV formation, and that the organization of VFCs occurred at a striking boundary between venous ECs that expressed ephrinB2 and those that did not. A conditional loss-of-function genetic approach has enabled us to show that both ephrinB2 and EphB4 were required for these early organizational events and that EphB4 was required for postnatal VV development.

\section{Results}

Patients with mutations in EPHB4 had fewer VVs and showed deep venous reflux. Pathogenic mutations in EPHB4 were recently described in 2 families with primary lymphatic-related fetal hydrops (LRFH), with autosomal dominant inheritance (21). Adults in both families had a notably early onset of lower limb venous disease. We therefore characterized the numbers of valves per vein in these patients $(n=5)$ and an unaffected relative using ultrasonography, and compared these results with a control population $(n=12$; Supplemental Table 1; supplemental material available online with this article; https://doi.org/10.1172/jci. insight.140952DS1). VVs were readily detected in the unaffected relative and other controls, but fewer VVs 
were detected in patients carrying a heterozygous mutation in EPHB4, including 3 patients with a mosaic mutation in $E P H B 4$ (fold change $0.2 \pm$ SD 0.29 for mosaic carriers, and $0.17 \pm 0.36$ for constitutive carriers, $P=1.7 \times 10^{-11}$, 1-way ANOVA, $F=30.3,2$ df; Figure 1, A and B, and Supplemental Figure 1). Ninety-two veins were analyzed in 13 controls, and 40 veins were analyzed in 5 mosaic or constitutive EPHB4 mutation carriers. Given the substantial loss of VVs in those with constitutive EPHB4 mutations, too few VVs were available for detailed analysis of leaflet length in constitutive mutation carriers, but those VVs that were identified were not significantly shorter than controls (Supplemental Figure 1; fold change $1.15 \pm 0.63$ for mosaic carriers, and $0.67 \pm 0.48$ for constitutive carriers, $P=\mathrm{NS}$ ). Groups were matched for age and sex $(P=\mathrm{NS})$. Those carrying an EPHB4 mutation had a mean popliteal reflux duration of 1.37 seconds, above the accepted diagnostic threshold of 1 second for severe deep venous reflux. Both patients with constitutive EPHB4 mutations exhibited a mean popliteal vein reflux duration of 1 second or longer (Figure 1, C and D, and Supplemental Figure 1).

EphB4 was expressed on E18 and PO and was required for normal VFC organization. EphB4 is the main ephrinB2 receptor in the vasculature, and these proteins often exhibit a complementary expression pattern during tissue segmentation $(13,35)$. Our analysis initially focused on embryonic day 18 (E18) and P0. We localized EphB4 expression in the region of the developing valve in Efnb2 ${ }^{G F P}$ mice and then examined whether EphB4 was required for organization of VFCs on P0.

On E18, when VFCs were in the process of organizing themselves at the site of developing valves, EphB4 expression appeared to be stronger immediately upstream of areas showing VFC organization and adjacent to VFCs with high Efnb2 expression (Figure 2A and Supplemental Figure 2C). Quantification of Efnb2 ${ }^{G F P}$ signal and EphB4 immunosignal across these organizing areas (yellow box in Figure 2A) confirmed relatively complementary expression with significantly higher EphB4 upstream and higher Efnb2 ${ }^{G F P}$ downstream of the VFCs (Figure 2B). Conversely, VFCs nearer the superior or inferior edges of the vessel already coexpressed Efnb2 $2^{G F P}$ and EphB4 (arrowheads in Figure 2A).

By P0, VFCs consistently reorientated and elongated to form a line of cells across the anterior femoral vein wall and partly extended across the posterior wall, defined as stage 1 of VV development (schematic in Figure 2A). Prior to this, development is described as stage 0. We had thought that EphB4 expression would be complementary to Efnb2 expression on P0, but EphB4 was immunolocalized variably throughout the valve region, with stronger expression within clusters of VFCs at the superior and inferior regions of the valve (Figure 2C, arrowheads), where we previously identified multiple proliferating VFCs (11). Coexpression of Ephb4 and Efnb2 was confirmed in Efnb2 ${ }^{G F P}$ mice (Figure 2D and Supplemental Figure 2B).

Deletion of Ephb4 on E15 resulted in disorganized VFCs on P0 (Figure 3A) albeit some VVs developed normally to stage 1 (Figure $3 \mathrm{~B}$ ).

Ephb4 was required for leaflet development to P6. We next localized the expression of EphB4 in VV leaflets on P6 and in adult mice. We then examined whether EphB4 is required for maturation of the valve leaflets up to P6.

EphB4 continued to be expressed in the endothelia of veins and VV leaflets on P6 and in adults (Figure $3 \mathrm{C}$, left panel). Expression was strongest on the lumen surface of the valve leaflet, including cells at the free edge of valve leaflet (Figure 3C, right panel). This expression is complementary to the previously identified lack of expression of Efnb2 in these free-edge cells (12). This could contribute to maintenance of their phenotype, which is clearly different to the rounded morphology of endothelia lining the sinus or lumen leaflet surfaces (12)

On P6 VVs are normally at stages 3 or 4 (schematic in Figure 3), which were defined, as previously, by the presence of 1 or 2 commissures (11). Deletion of Ephb4 on P0 led to a complete failure of valve leaflet formation by P6, with only a few Prox1-expressing or Foxc2-expressing cells remaining (Figure 3, D and E). This phenotype (Ephb4 deletion on P0, analyzed on P6) was more consistent and severe than deletion on E15, analyzed on P0 (Figure 3A).

Similar to other gene-deletion studies resulting in loss of VFCs by P6, there was an associated failure to establish a local reduction in the density of smooth muscle cells (SMCs) around the valve (Figure 3D; ref. 11)

VFC organization occurred at a developing boundary between ECs expressing and not expressing Efnb2. To visualize the Efnb2 expression pattern during VFC organization, we visualized the site of VV formation in the proximal femoral vein using confocal microscopy of wholemount samples from Efnb2 ${ }^{G F P}$ reporter mice (Figure 4A). Efnb2 ${ }^{G F P}$ signal was strong in femoral artery ECs (Figure 4A), and generally absent or at very low levels in venous endothelia in all samples analyzed, similar to previously reported findings (36, 37). Expression of Efnb2 by venous smooth muscle $\alpha$-actin-expressing mural cells was not detected (data not shown). Global 
A

음

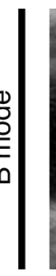

Unaffected relative
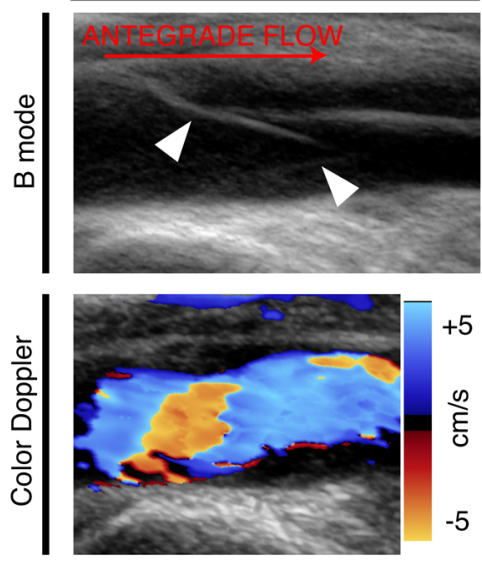

B

B
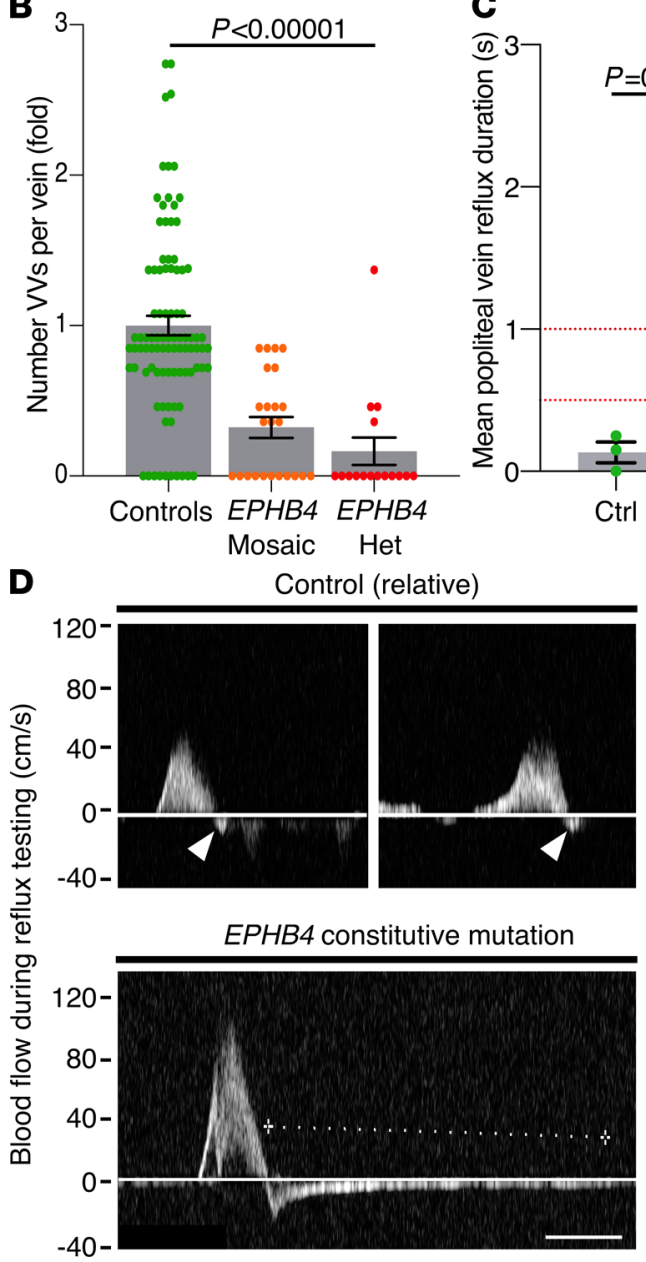

EPHB4 mutation
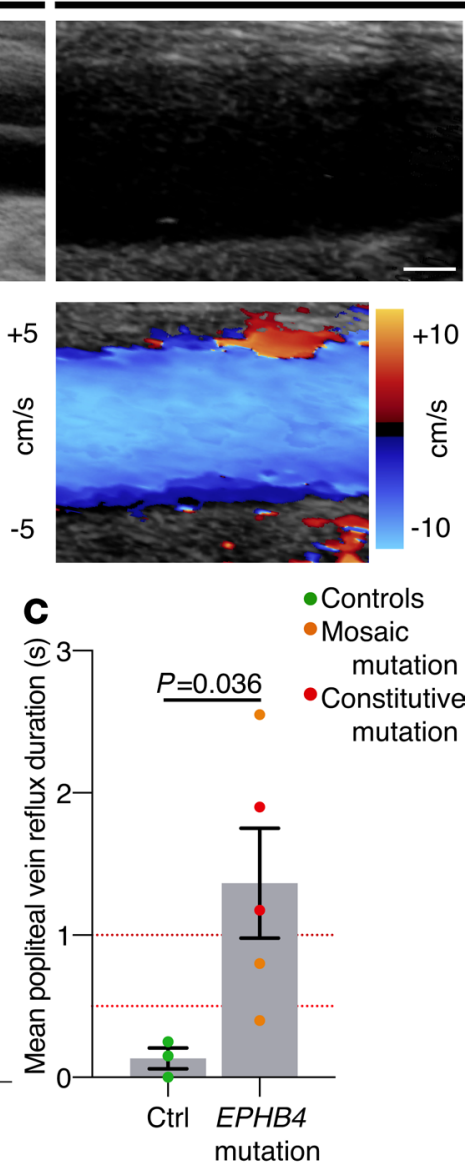

Controls

Mosaic mutation

Constitutive mutation
Figure 1. EPHB4 mutations cause human VV failure. (A) VVs (arrowheads) were readily identifiable in the veins of controls, including an unaffected relative, but were rare in patients with a mutation in EPHB4. B-mode and color Doppler images are shown of the popliteal vein. Blood flow left to right, velocity indicated by color scale. Scale bar: $2 \mathrm{mM}$. (B) Fewer VVs per vein were seen in participants with mosaic or constitutive (heterozygous) EPHB4 mutation $\left(P=1.7 \times 10^{-11}, 1\right.$-way ANOVA). $n=92$ veins in 13 controls, and 40 veins in 5 patients with $E P H B 4$ mutation (mosaic or constitutive). Data points represent individual veins. (C) Popliteal (deep) venous reflux was identified in mosaic and constitutive carriers of EPHB4 mutations $(P=0.036$, Mann-Whitney $U$ test). Blood velocity $\geq 0.5$ second indicates reflux and $\geq 1$ seconds indicates severe reflux (red dotted lines). Data points represent mean of left and right popliteal reflux duration for each individual. (D) Representative blood velocity in the popliteal vein during reflux testing is shown for an unaffected relative (with no significant reflux, arrowheads) and a patient carrying an EPHB4 mutation, demonstrating significant deep venous reflux (dotted line $=2.14$ seconds). Scale bar: $500 \mathrm{~ms}$ ). Throughout all figures, antegrade blood flow is from left to right. Data are shown as mean \pm SEM. VVs, venous valves; Het, heterozygous.

heterozygous knockout of Efnb2 (in the Efnb2 ${ }^{G F P}$ reporter) did not prevent development of stage 1 VVs by P0 ( $P=$ NS versus WT littermates, $n=32$ Efnb2 ${ }^{\text {GFP/wt }}$ VVs analyzed). On E18 the patterning of Prox $1^{\text {hi }}$ VFCs within the valve-forming region was more variable than on $\mathrm{P} 0$, with areas of Prox $1^{\text {hi }}$ cells (e.g., the superior but not inferior area) showing organization (i.e., reorientation and elongation of cells; Figure 4A, upper panel versus lower panel). The organizing VFCs, and endothelia just downstream of organizing VFCs, expressed Efnb2 (Figure 4A, green box), whereas areas without VFC organization did not develop a boundary in ephrinB2 expression (Figure 4B, blue box). Quantification of the Efnb2 ${ }^{G F P}$ signal on E18 confirmed a boundary in expression of Efnb2 in regions of organized cells, but not in adjacent nonorganized regions (Figure 4B). 
A

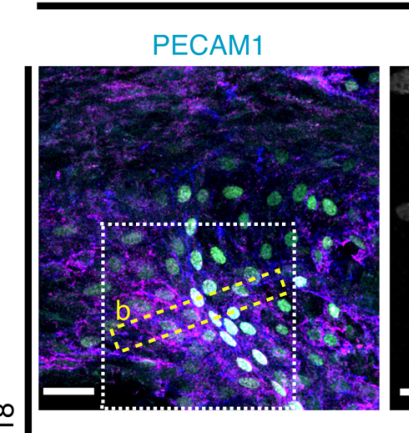

Efnb2 $2^{\text {GFP/wt }}$

$\frac{\infty}{\square}$

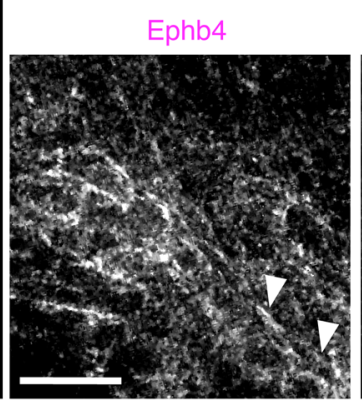

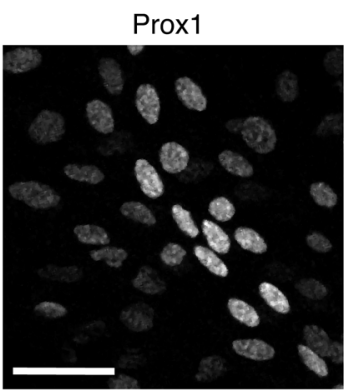


Relative

fluorescence

intensity Efnb2 ${ }^{\mathrm{GFP}}$ (nuclear)

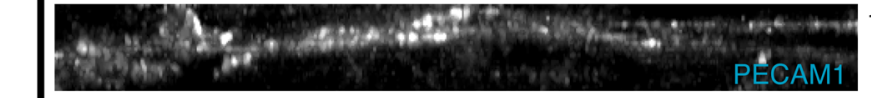

$\stackrel{\infty}{\square}$
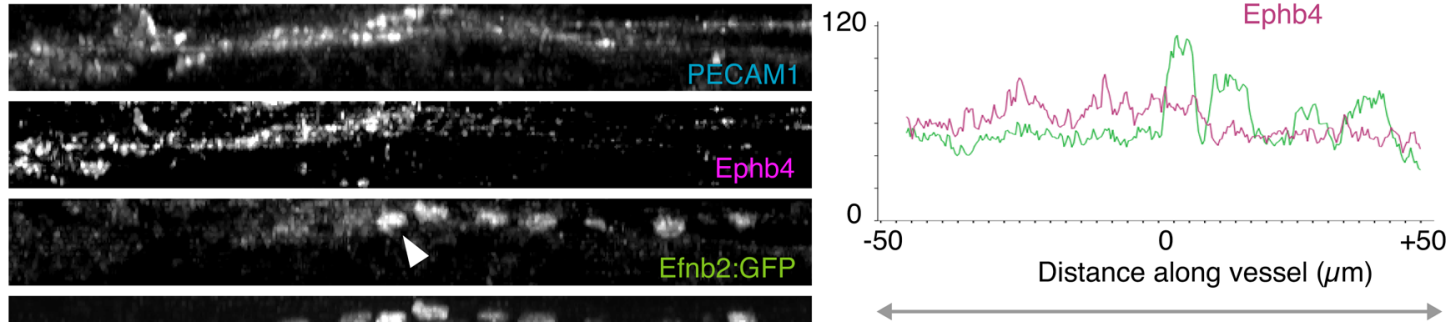

Ephb4

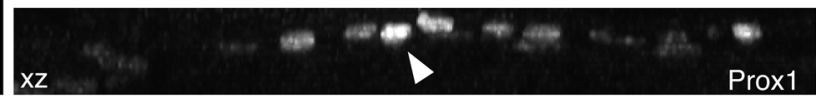

upstream

downstream

C

WT

\section{D}

Efnb2 $2^{G F P / w t}$


Figure 2. Expression of EphB4 in Efnb2GFP reporter E18 and PO. (A) Localization of PECAM1 (blue), Ephb4 (magenta), Prox1 (white), and Efnb2:GFP (green) in heterozygous Efnb2 ${ }^{\mathrm{CFP}}$ mice on E18. Part of an E18 VV is shown, and the white boxed area (which contains organizing VFCs) is shown enlarged in single channel images. Only the anterior vein wall is shown. Arrowhead indicates a VFC nearer the inferior edge of the vessel coexpressing Ephb4 and Efnb2GFP. The schematic indicates stages 0 and 1 of VV development, as previously defined in ref. 11. Red $=$ Prox ${ }^{\text {hi }}$ VFCs, which form a continuous line across the anterior vein wall at stage 1. The orientation of all confocal $Z$ stacks is indicated and is the same throughout all figures. (B) An XZ projection (13.6- $\mu \mathrm{m}$ deep) and the fluorescence intensity profile for Efnb2CFP and EphB4 are shown across the organizing VFCs, indicated by the yellow boxed area in $\mathbf{A}$. The EphB4 signal is stronger upstream (to the left) of the VFCs (indicated by arrowheads, or " 0 " on the graph $\mathrm{x}$ axis), whereas the Efnb2GFP signal is stronger in VFCs and downstream ( $P$ $<0.0001, n=6$ VVs, 2-tailed $t$ test). The multichannel image does not include Prox1. (C) In WT VVs on P0, Proxh ${ }^{\text {hi }}$ VFCs expressed EphB4, and it was particularly strongly expressed in the superior and inferior areas of the vein (arrowheads). (D) Coexpression of Ephb4 and Efnb2 was confirmed in Efnb2GFP mice. Z projections $(6 \mu \mathrm{m})$ of the upper and lower regions of a valve are shown. Arrowheads indicate reorientated VFCs (orange). (Uncropped images are provided in Supplemental Figure 2B.) Scale bars: $20 \mu \mathrm{m}$. VFCs, valve-forming cells; VVs, venous valves; E18, embryonic day 18; P0, postnatal day 0. 
A

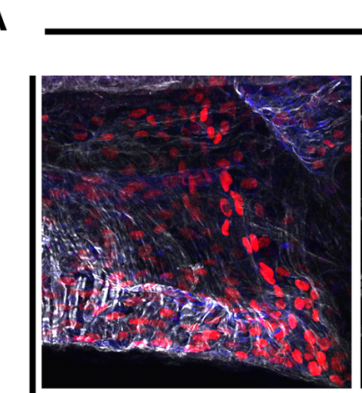

인

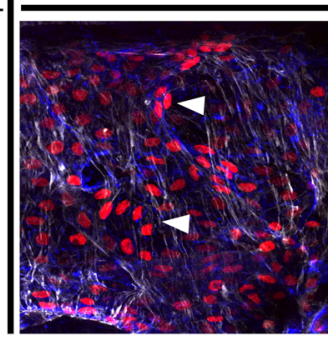

C

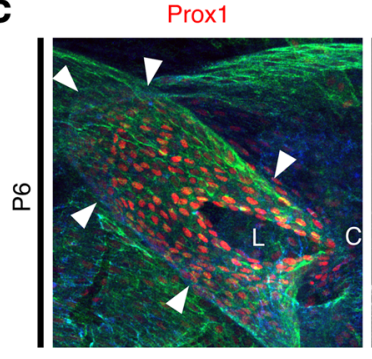

Control

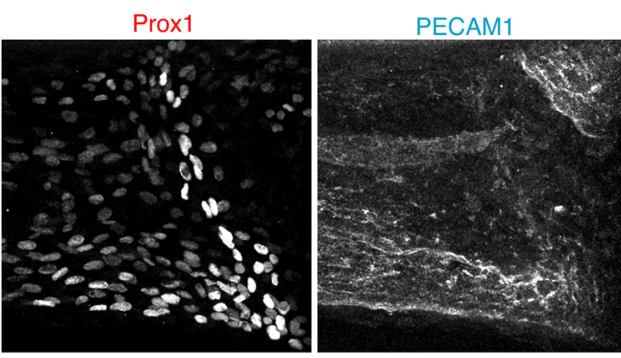

Ephb4/x/x



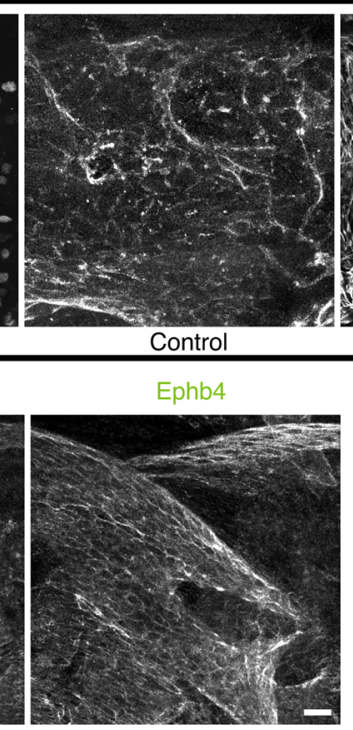
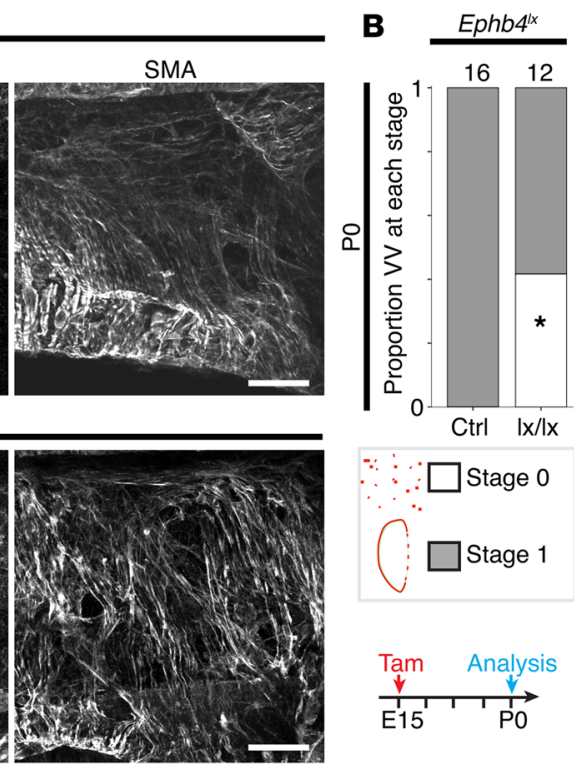

$\because \square$ Stage 0

$\cap \square$ Stage 1



D

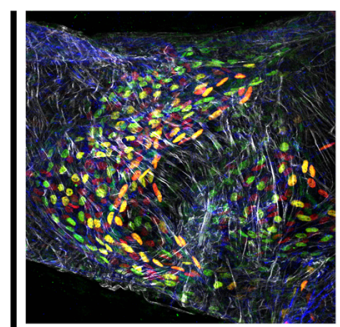

$\therefore$

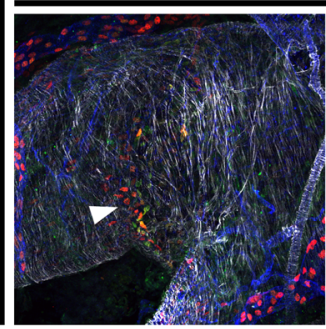

PECAM1

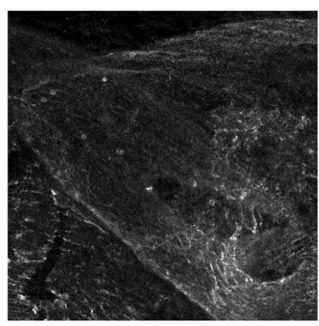

Control

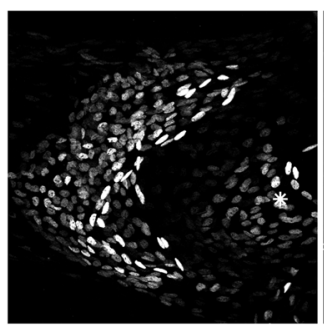

Foxc2

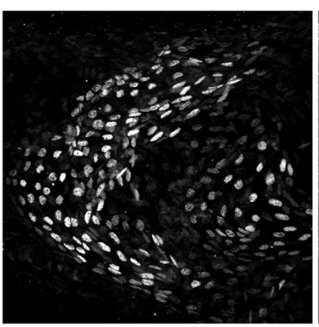

PECAM1


Ephb4 $4^{\mid x / x}$

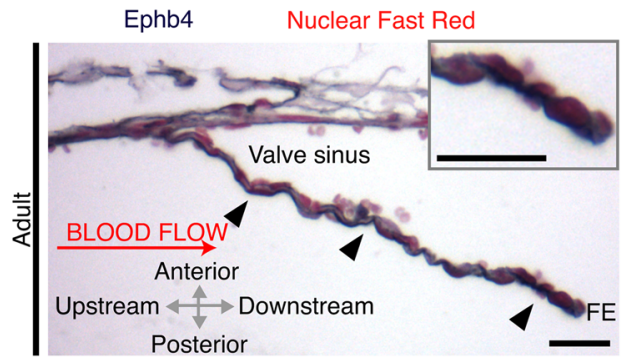

SMA
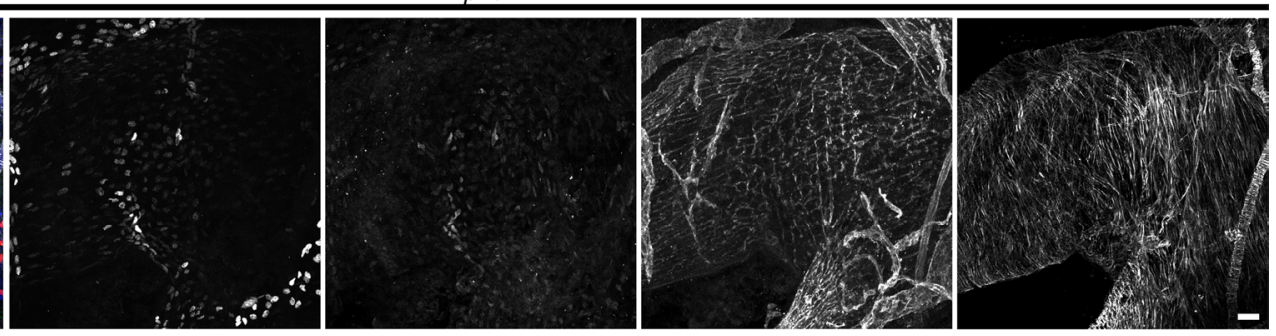

E

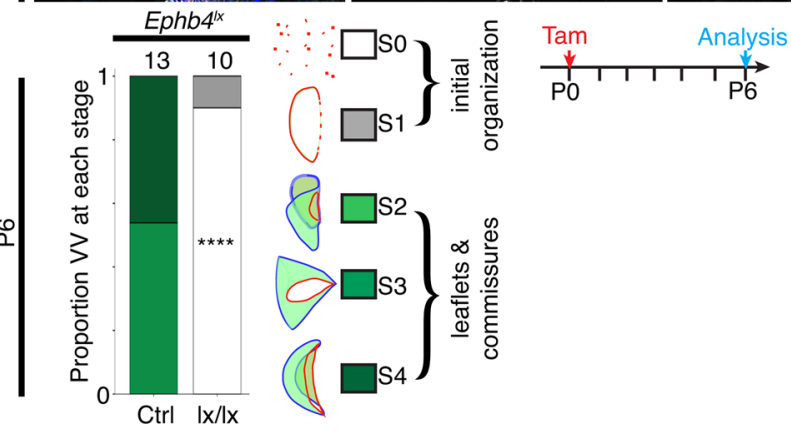


Figure 3. EphB4 is expressed on E18 and PO and is required for normal VFC organization and leaflet development to P6. (A and B) Homozygous deletion of Ephb4 on E15 (analyzed on PO) resulted in disrupted organization of VFCs, similar to deletion of Efnb2, albeit some VVs appeared to develop normally. The number of VVs analyzed for each condition is indicated above each bar in the chart. ${ }^{*} P=0.008$, Fisher's exact test. Scale bars: $20 \mu \mathrm{m}$. (C) EphB4 was localized in WT P6 VVs and surrounding vein. The leaflet of a stage 3 VV is indicated by arrowheads. $L=$ valve lumen and $C=$ the single commissure. In adult VVs, longitudinal sections were prepared, and EphB4 (dark blue stain) was most strongly localized to the luminal surface of VV leaflets (black arrowheads) and leaflet free edge (enlarged in inset). The counterstain is Nuclear Fast Red. Arrows indicate the orientation of the adult histological section only (all confocal images are oriented as shown in Figure 2). (D and E) Induction of homozygous Ephb4 deletion on PO with tamoxifen (analysis on $\mathrm{P6}$ ) resulted in entirely absent VV leaflets and failure to remodel the surrounding SMCs (arrowheads in upper panel) on P6. Only a few Prox $7^{\text {hi }} /$ Fox$\mathrm{c} 2{ }^{\mathrm{hi}}$ cells remained (arrowhead in lower panel). The asterisk indicates a downstream tributary valve. (E) Bar chart shows the proportion of VVs identified at each stage, with stage and color indicated in adjacent key, on P6 for the indicated genotypes. The number of VVs analyzed for each condition is given above each bar. ${ }^{* * *} P<0.00005, \chi^{2}$ vs. control, $n=13$ control VVs vs. 10 Ephb4 deleted. Scale bars in A, C, and D: $20 \mu \mathrm{m}$. VFCs, valve-forming cells; VVs, venous valves; E18, embryonic day 18; P0, postnatal day 0; Tam; tamoxifen; SMA, smooth muscle $\alpha$-actin; SMC, smooth muscle cell.

On P0 Efnb2 was consistently expressed (and more strongly than on E18) by the line of Prox $1^{\text {hi }}$ VFCs and in cells downstream, but not upstream, of the VFCs (Figure 4A, lower panel). Quantification confirmed the boundary in Efnb2 ${ }^{G F P}$ signal, with a peak in Efnb2 expression coinciding with Prox $1^{\text {hi }}$ VFCs (Figure 4C). Whereas on E18 the downstream Efnb2 ${ }^{G F P}$ signal was marginally higher than the upstream signal (Figure 4, A and B), on P0 this difference was more marked (Figure 4, A and C). These results suggest that the Efnb2 expression boundary was formed concomitantly with the organization of Prox $1^{\text {hi }}$ VFCs and suggest that an Eph-ephrin interaction within venous endothelia might have participated in the regulation of VFC organization.

Analysis of this valve-forming region on P0 in WT mice by transmission electron microscopy (TEM) demonstrated that development of the core of the valve leaflet is more advanced than previously characterized, with the presence of interstitial cells within the leaflet, which is already protruding from the vessel wall (Figure 4D, upper panel; ref. 11). VFCs at the leading edge of the protruding leaflet were partly detached from the underlying basement membrane, consistent with their progressive reorientation and migration (Figure 4D, upper panel, arrowheads), which has been previously identified in developing LV (48). TEM analysis on P6 and in adult mice confirmed the presence of interstitial cells in murine VV (Figure 4D, middle and lower panels, and Supplemental Figure 3, A-C), consistent with their known presence in, for example, rabbit VV (2). The presence of interstitial cells in human VV was confirmed by TEM and histology (Supplemental Figure 3, D and E). Connexin43 (Cx43) and Connexin47 (Cx47), proteins implicated in human VV disease (11), were immunolocalized to human VV interstitial cells (Supplemental Figure 3F).

Efnb2 was required for normal VFC organization. Having established the expression pattern of Efnb2 during VFC organization, we then examined whether Efnb2 is required for the organization of VFCs on P0. We performed conditional gene deletion using floxed Efnb2 alleles and Prox1Cre ${ }^{E R T 2}$, and quantified each valve according to developmental stage and also quantified the elongation and reorientation of Prox $1^{\text {hi }}$ cells (as previously described in refs. 11, 12, 48). Heterozygous deletion on E15 did not significantly affect VV development to stage 1 (Figure 5A, middle panel, and Figure 5B). There was, however, a small but significant reduction in VFC nuclear elongation (Figure 5, C and D), but no difference in their reorientation (Figure 5, E and F). Homozygous deletion of Efnb2 resulted in disorganized VFCs that failed to reach stage 1 of development on P0, with a similar pattern of disorganization to that seen with deletion of Ephb4 $(P<$ 0.001; Figure 5A, lower panel, and Figure 5B). Prox $\mathrm{h}^{\mathrm{hi}}$ cells were present but appeared to be distributed across a wider upstream-downstream region of the vessel, and exhibited markedly reduced elongation (Figure 5, C and D; $P<0.00005)$ and reorientation $(P<0.005$; Figure 5, E and $\mathrm{F})$. These findings demonstrate that endothelial Efnb2 was required for the normal organized patterning of VFCs on P0.

These results, together with those we described for Ephb4, show that the expression of EphB4 and $E f n b 2$ was dynamic during VV organization, and complementary expression (Efnb2 higher downstream, EphB4 higher upstream) occurred during the process of organization on E18, but by P0, VFCs expressed both Efnb2 and EphB4.

Efnb2 was required for projection of VFCS into the vessel lumen, normal expression of integrin $\alpha 9$, and normal polarity. We prepared longitudinal semithin sections in the XZ-plane of the wholemount preparations, to more clearly examine projection of VFCs into the vessel lumen. Compared with littermate controls, VFCs failed to project into the vessel lumen in homozygous Efnb2-deleted cells (Figure 6, A and B). We hypothesized that failure to correctly express integrin $\alpha 9$ could be a mechanism underlying the failure of VFCs to organize and project into the lumen in Efnb2-deleted mice, because integrin $\alpha 9$ is required in valve formation 
A

Efnb2 GFP/wt


Figure 4. Formation of ephrinB2 expression boundary in VV-forming region. (A) Localization of PECAM1 (blue), Prox1 (red), and Efnb2GFP reporter signal (green, His-tagged and therefore nuclear) on E18 and PO in heterozygous Efnb2GFP mice. Wholemount preparation of the proximal femoral vein is shown. On E18 there was partial, and variable, organization of VFCs, for example, in the superior area of the VV-forming region but not the inferior area. Those areas with organization on E18 showed a weak Efnb2GFP expression boundary, which was clearer on PO (white arrowhead). Dotted lines indicate the femoral vein boundary, adjacent to the femoral artery. As expected, arterial endothelial cells showed stronger Efnb2GFP signal. * Indicates an overlying arterial branch (cut). (B and C) On E18, analysis of the relative fluorescence intensity across developing valves revealed a peak in Efnb2GFP signal (green line) coincident with that of Prox $1^{\text {hi }}$ (red) VFCs in organizing areas, but not in adjacent areas that are not yet organized (blue line). At both E18 and P0, Efnb2GFP signal is stronger downstream, and this difference is more apparent on P0. Mean of 6 VVs and 7-12 regions analyzed per VV and representative regions analyzed are shown boxed (green, blue) in A. Ps in B and C are 2-tailed $t$ tests comparing Efnb2GFP proximal and distal to the VFC leading edge. NS. (D) TEM analysis on PO showed rotated VFCs detached from underlying basement membrane (arrowheads). Interstitial cells $\left(^{*}\right)$ populated the developing leaflet core, and persisted on $\mathrm{P} 6$ and in adults. TEM micrographs are orientated at $90^{\circ} \mathrm{C}$ 
to confocal images, as indicated by arrows on PO in D. Further examples of interstitial cells (in murine and human VVs) are shown in Supplemental Figure 3. $n \geq 6$ VV and blood flow left to right at all time points and in B and C. Scale bar in A is $20 \mu \mathrm{m}$ and scale bar in $\mathbf{D}$ is $2 \mu \mathrm{m}$ on P0-P6, 500 nm in adults. VFCs, valve-forming cells; VVs, venous valves; E18, embryonic day 18; P0, postnatal day 0; TEM, transmission electron microscopy.

for extracellular matrix remodeling, and for VV leaflet growth and maintenance $(11,12,49)$. On P0 integrin $\alpha 9$ expression was largely localized to the line of VFCs on the anterior vein wall (Figure 6C, upper panel). After Efnb2 deletion, the integrin $\alpha 9$ expression pattern followed the abnormal, broader distribution of the Prox $1^{\text {hi }}$ cells, and appeared haphazard (Figure 6C), likely precluding normal matrix remodeling.

Because VFCs appeared in a broader region after Efnb2 deletion, we hypothesized that without guidance from ephrin-Eph interactions on E18, these cells would be disorientated on P0. In LV formation, lymphatic ECs elongate and migrate centrally from the edges of the vessel $(48,50)$ and in migratory ECs, the Golgi apparatus is positioned apically of the nucleus (51). We therefore analyzed VFC alignment by costaining for a Golgi marker, and examined the alignment of cells with the forming VV structure (Figure 6D, upper panel). In littermate controls on P0, cells were consistently aligned across the vessel anterior wall, whereas in all samples with homozygous Efnb2 deletion, there was a disrupted pattern (Figure 6D, lower panel).

Gap junction intercellular communication and proliferation. Expression of ephrinBs may regulate cell behavior by modulating connexin communication domains, including via Cx43 $(32,46)$. Cx43 and Connexin37 (Cx37) have highly regulated expression patterns around VFCs on P0, and both are required for venous, lymphatic, and lymphovenous valve formation $(11,13,14,52-54)$. Large gap junction plaques containing Cx37 are normally expressed by Prox ${ }^{\text {hi }}$ VFCs on $\mathrm{P} 0$, whereas $\mathrm{C} x 43$ is primarily expressed in a region just upstream of the organized VFCs on P0. Homozygous deletion of Cx43 (using Prox $1 C r e^{E R T 2}$ ), or homozygous knockout of $\mathrm{Cx} 37$, results in a failure of organization of VFCs on P0, which is reminiscent of the phenotype seen with homozygous deletion of Efnb2 $(11,13,14)$. This failure of organization on P0 is followed by complete loss of valve structure (13). We therefore examined the expression patterns of Cx37 and Cx43 relative to Efnb2 expression in the Efnb2 ${ }^{G F P}$ reporter mice and after homozygous deletion of Efnb2. The normally highly restricted expression patterns of Cx37 and Cx43 were disrupted on P0 after homozygous Efnb2 deletion (Figure 7, A and B). In the Efnb2 ${ }^{G F P}$ reporter, $\mathrm{Cx} 37$ localization indicated gap junction plaque formation around Efnb2-expressing VFCs (Figure 7A, white arrowheads in upper panel), whereas after Efnb2 deletion, no plaque formation was identified (or possibly plaques were very much smaller), and Cx37 expression appeared more widespread in the region of the VFCs (Figure 7B).

In previous genetic loss-of-function experiments (including knockout of Cx37), disruption of VFC organization was associated with a reduction in VFC proliferation (11), and so we next examined whether deletion of Efnb2 altered VFC proliferation or apoptosis. As previously described, $\mathrm{Ki}^{-} 7^{+}$-proliferating VFCs appeared more abundant in the superior and inferior regions of the valve on P0 (11). A reduction in the proportion of proliferating VFCs was seen after Efnb2 deletion $(P<0.001$; Figure 7, C and D), but no effect on apoptosis (as detected by Caspase-3 expression) was observed (data not shown).

\section{Discussion}

We have identified human VV failure and deep venous reflux caused by mutations in EPHB4. This phenotype was more severe (i.e., a greater loss of valves) than that previously identified in patients with mutations in FOXC2 or GJC2 (a fold change versus controls of $0.2 \pm \mathrm{SD} 0.29$, mosaic EPHB4, or $0.17 \pm$ 0.36 , constitutive heterozygous $E P H B 4$, for the reduction in mean VVs per vein; ref. 11). Almost all of these patients did not have clinical evidence of chronic lower limb primary lymphedema. Some presented with nonimmune fetal hydrops, which was of lymphatic origin, but it had resolved soon after birth. After that, their most obvious clinical sign of disease was early onset prominent or varicose veins, and venous insufficiency (21). We now know that this is venous valvular aplasia, and therefore mutations in EPHB4 should be considered as a cause of primary venous valvular aplasia $(5-8,21,55)$. Dysfunction of the deep VVs increases the rate of progression of chronic venous insufficiency, with a higher rate of chronic venous ulcer formation. The management of deep venous reflux is extremely challenging, because currently there are no reliably effective therapies beyond invasive surgical construction of neovalves (3)

Heterozygous mutations in EPHB4 are reported to cause CM-AVM2, vein of Galen aneurysmal malformation, LRFH, and central conducting lymphatic anomaly (CCLA), but the mechanisms underlying these different presentations remain unclear $(21,22,25,26,28)$. The clinical descriptions of patients with a lymphatic phenotype such as LRFH and CCLA also include clear features of venous 
A

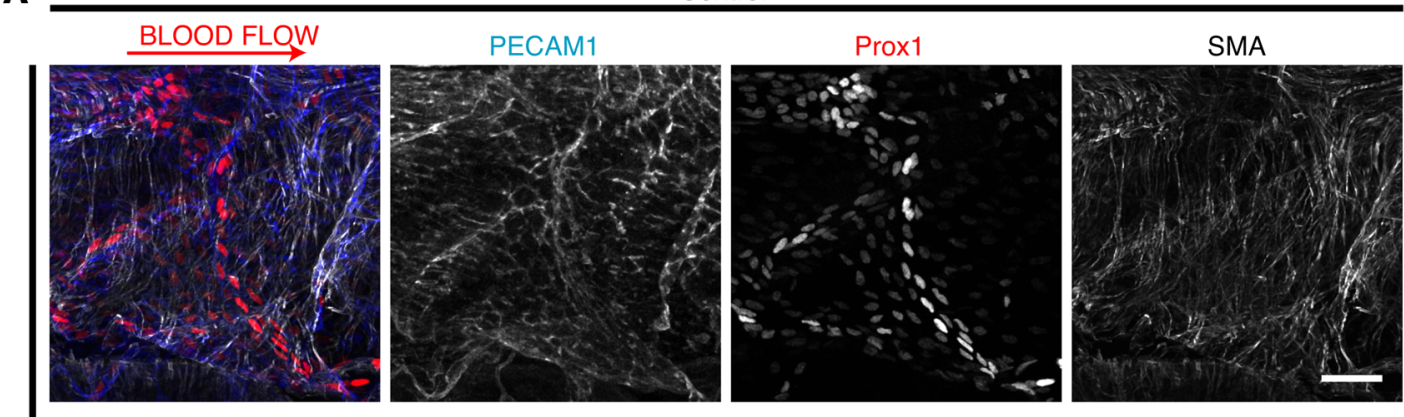

Efnb2/x/mt
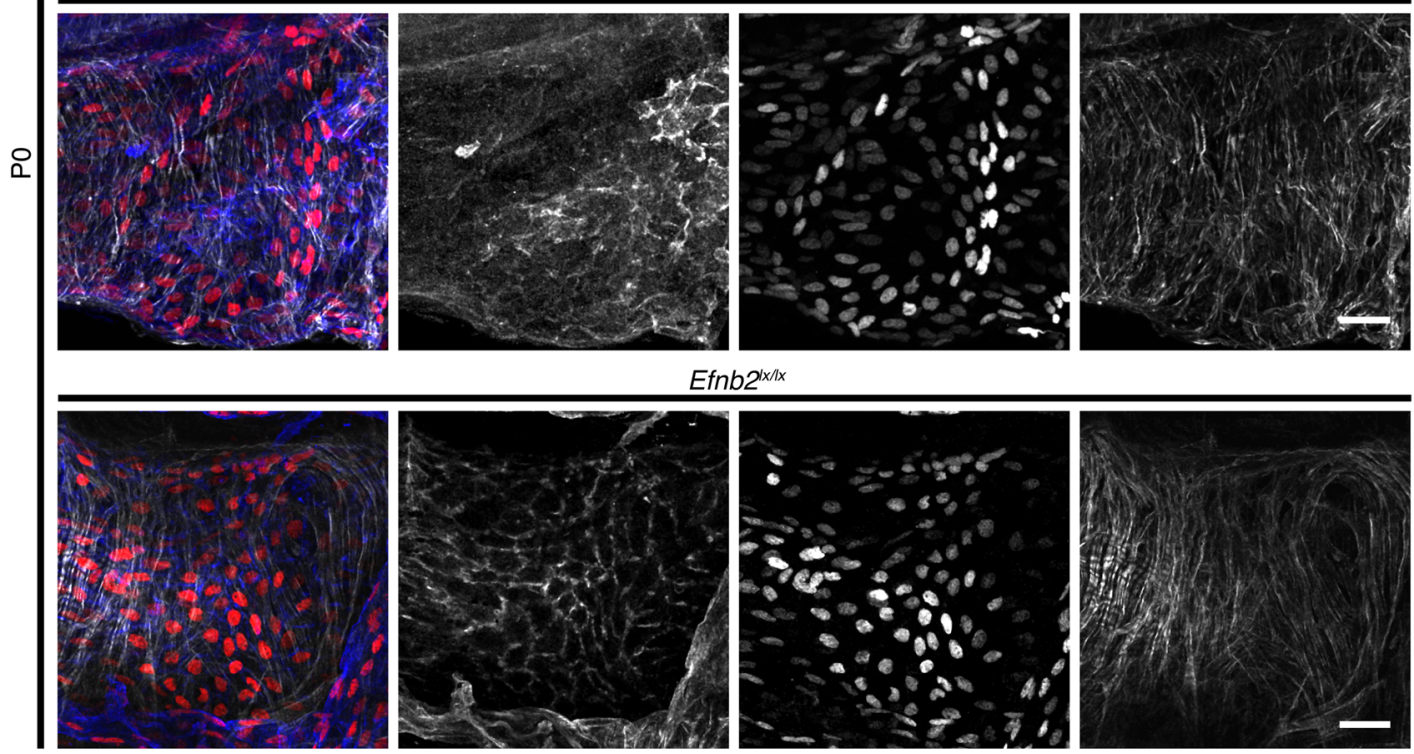

Efnb2/x/x


c
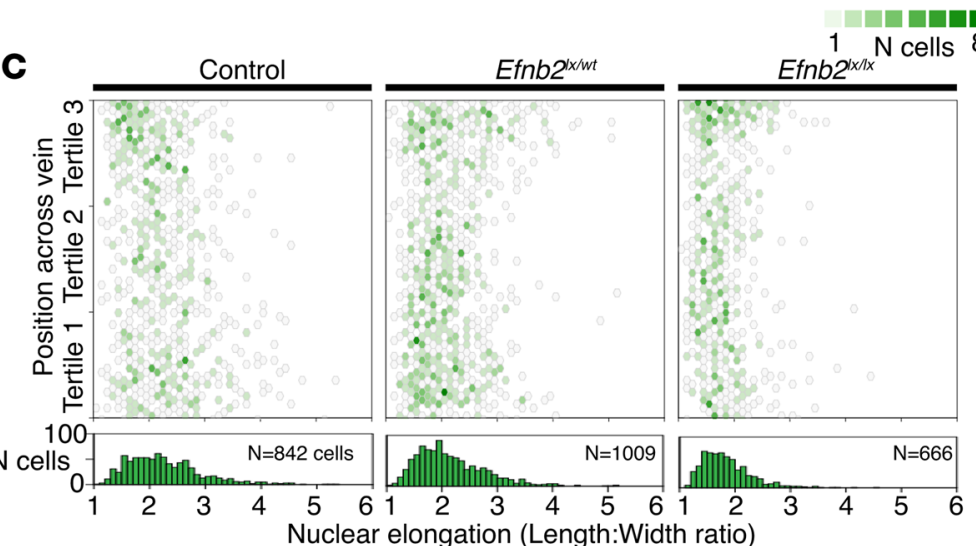

$\mathbf{E}$

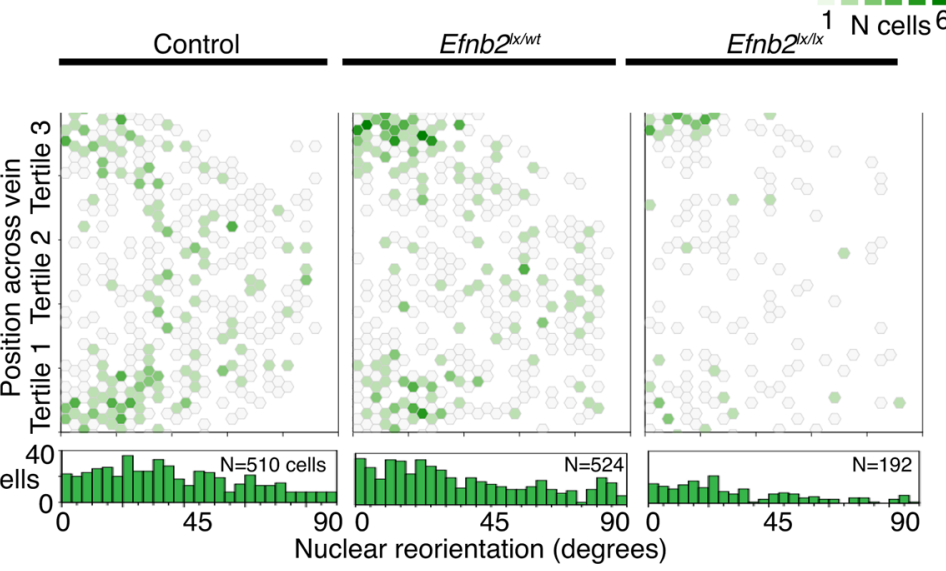

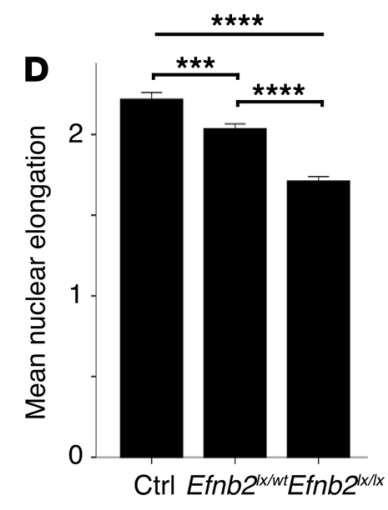

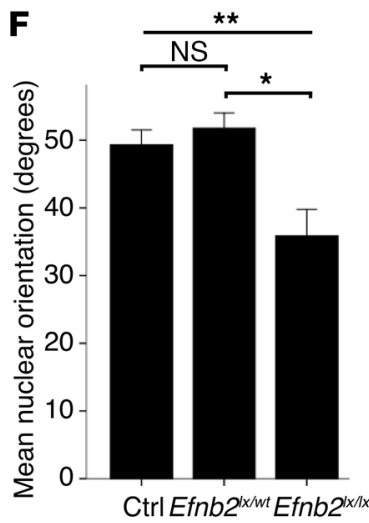

B

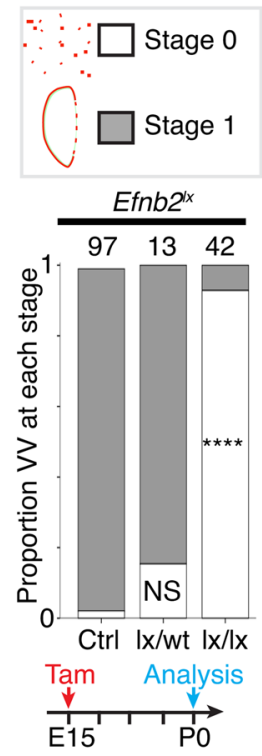


Figure 5. Effect of Efnb2 deletion on organization of VFCs. (A and B) Localization of PECAM1 (blue), Prox1 (red), and SMA (white) in littermate controls and heterozygous (Efnb2 ${ }^{l x / w t}$ ) and homozygous (Efnb2lx/Ix) mice on P0, after tamoxifen induction of Efnb2 deletion on E15. In controls and Efnb2 ${ }^{1 x / w t}$ mice, valves reached stage 1 of development, as normal. Homozygous deletion resulted in a failure to organize normally, with Prox $1^{\text {hi }}$ cells distributed over a wider upstream-downstream area of the vein and failure of VFCs to elongate and reorientate. (B) The bar chart shows the proportion of VVs identified at stage 0 (white) and stage 1 (grey) on PO for the indicated genotypes, and the number of VVs analyzed for each condition is given above each bar. Ps derive from 2-sided Fisher's exact test vs. control. (C) Hex-binned scatterplot of VFC elongation (length/width ratio) across the vein from superior to inferior. $n=2517$ cells, $\geq 6$ VVs. (D) Bar chart ( \pm SEM) summarizing the results from C showing that both heterozygous and homozygous deletion resulted in significant reductions in VFC elongation. One-way ANOVA with Bonferroni's post hoc test. For between groups 1-way ANOVA, F = 109 with $2 \mathrm{df}, \mathrm{P}=3.2 \times 10^{-46}$. (E) Hex-binned scatterplot of VFC reorientation (in VFCs with nuclear length/width ratio $\geq 2$ ) across the vein from superior to inferior. $n=1226$ cells, $\geq 6$ VVs. After homozygous deletion, the VFCs with correctly reorientated nuclei were lost, particularly in the center of the vessel. (F) Bar chart ( \pm SEM) summarizing the results from E. Homozygous deletion resulted in significantly reduced reorientation. One-way ANOVA with Bonferroni's post hoc. For 1 -way ANOVA, $F=7.1$ with $2 \mathrm{df}, P=0.0009 .{ }^{*} P<0.05,{ }^{*} P<0.005,{ }^{*}{ }^{*} P<0.0005$, ${ }^{* * * *} P<0.00005$. Scale bars: $20 \mu \mathrm{m}$. VFCs, valve-forming cells; VVs, venous valves. SMA, smooth muscle $\alpha$-actin.

disease such as varicose veins, venous hypertension, or venous reflux. Similar to the cases presented, it seems likely that the patients reported by Li et al. may also be affected by VV aplasia and deep venous reflux (considering their increased lower limb pigmentation and venous stasis; ref. 25). The early age at onset of clinical signs of venous insufficiency (for example, varicose veins, hemosiderin deposition) in affected individuals, and the near absence of VVs in the scanned veins of affected children observed here, is consistent with a failure of VV formation, rather than early degeneration. These features are not described for CM-AVM2, or vein of Galen aneurysmal malformation, and it is unclear whether the mutations causing these syndromes will also cause VV defects $(22,26)$. EphrinB2 is required for normal $\mathrm{CV}$ formation in mice, but no CV defects were noted on echocardiography in the patients reported here, or those reported elsewhere $(21,25,28)$. It remains unclear how, in the settings of developmental blood vessel formation and in the adult capillary bed, ephrinB2-EphB4 interaction leads to specification and subsequent maintenance of arterial and venous endothelia, yet both are expressed in mature veins to regulate the formation of valves $(12,22,23,38,41)$. Further work is needed to delineate the context and maturation-dependent regulation of these endothelia.

Previous in vitro analysis of the EPHB4 mutations studied here (p.Arg739Glu and p.Ile782Ser) demonstrated that they exhibit greatly reduced kinase activity, but do not exert a dominant negative effect on the expression of WT EPHB4 protein (21). Any effect on WT EPHB4 activity is unknown. The ratio of ephrinB2 to EphB4 expression is disturbed at both mRNA and protein level in ECs cultured from patient arteriovenous malformations, with greatly reduced EphB4 expression compared with a control cell line (56). The mutant EPHB4 protein implicated in CM-AVM2 becomes trapped in vesicles $(22,28)$, whereas that implicated in LRFH is presented on the cell membrane (28), but the exact signaling implications of these findings are yet to be elucidated. The requirement for ephrin/Eph signaling at multiple stages of VV development and maintenance complicates any attempt to develop molecular therapy aiming to directly restore valve function. It is possible that pharmacological stimulation or inhibition of the pathway downstream of EPHB4 might be helpful to overcome the resulting aberrant signaling $(22,25)$

The extent of overlap of the genetic causes of VV failure and varicose veins is unclear since regulation of VVs is understudied, but some important indications of similarity have already emerged, including the identification of PPP3R1 and PIEZO1 in genome-wide association studies of varicose veins, and in mice as critical regulators of VV development $(11,57,58)$. Delineating the roles of the various genes implicated in VV pathogenesis is important and may lead to novel therapies, which could be targeted toward patients at risk of deterioration to chronic ulceration (59)

In this study we have identified a striking "boundary" in the endothelial expression of Efnb2 at the site of developing VVs (meaning a demarcation between ephrinB2 ${ }^{\text {lo }}$ upstream cells and ephrinB2 ${ }^{\text {hi }}$ VFCs and cells immediately downstream), and that both ephrinB2 and EphB4 are required for normal organization of VFCs at this critical stage of development in mice. Because ephrinB2 remains the only known ligand for EphB4, this leads us to speculate that an ephrinB2-EphB4 interaction within venous endothelia regulates VV formation. We also show that EphB4 is required for VV maturation. On E18, in areas where VFCs appeared to be in the process of reorientating to become transversely aligned, EphB4 expression was stronger just upstream of the ephrinB2-expressing VFCs. We speculate that at this time point, EphB4 ${ }^{\text {hi }}$ regions upstream from VFCs may be acting to repel ephrinB2 ${ }^{\text {hi }}$ VFCs, guiding them to reorientate to lie transversely across the vessel to form a line across the anterior of the lumen. We were unable to localize ephrinB2 because of a lack of specific antibodies, and this inability to colocalize EphB4 and ephrinB2 is a limitation 
A

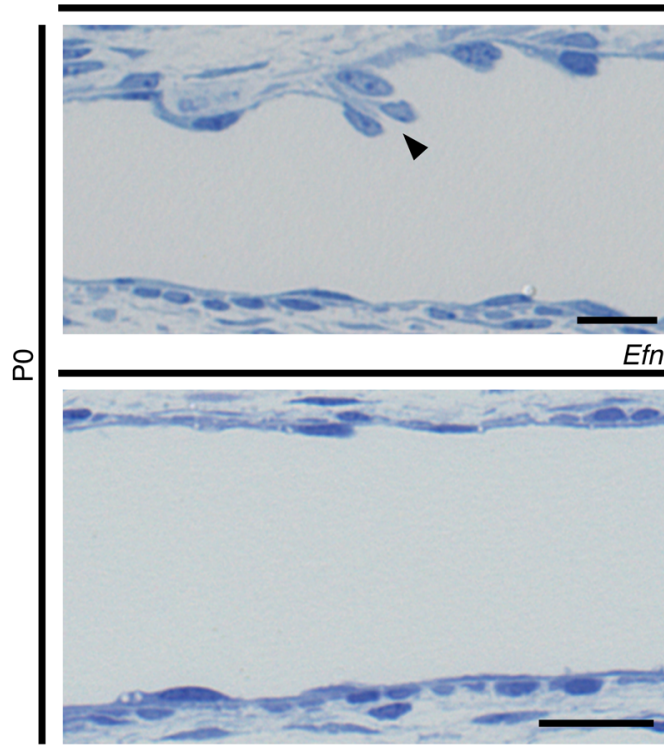

C

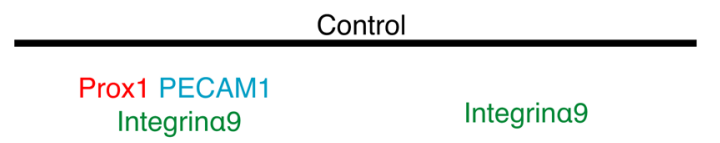

Control



$f n b 2^{x / 1 x}$

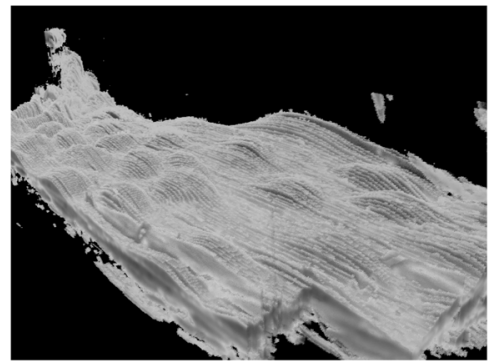

D
Anterior

Upstream $\stackrel{4}{\longrightarrow}$ Downstream

Posterior

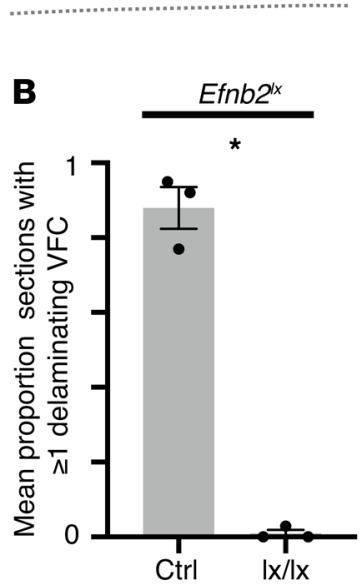

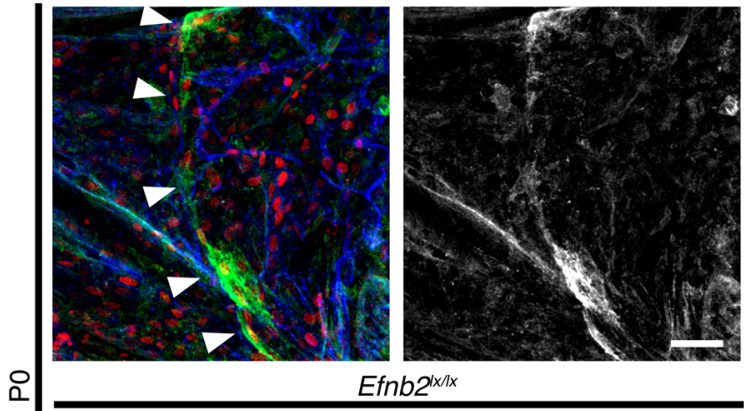

Prox1 Golgi PECAM1

Control

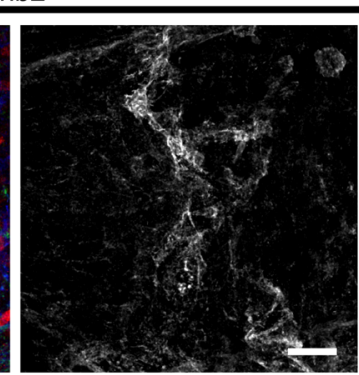

ㅇ
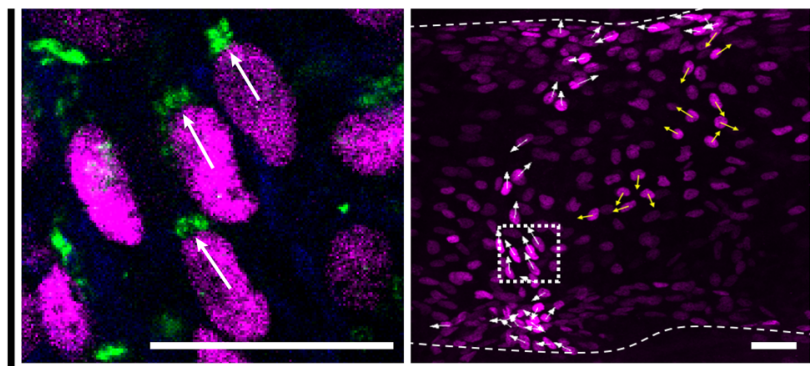

$E f n b 2^{1 x / 1 x}$
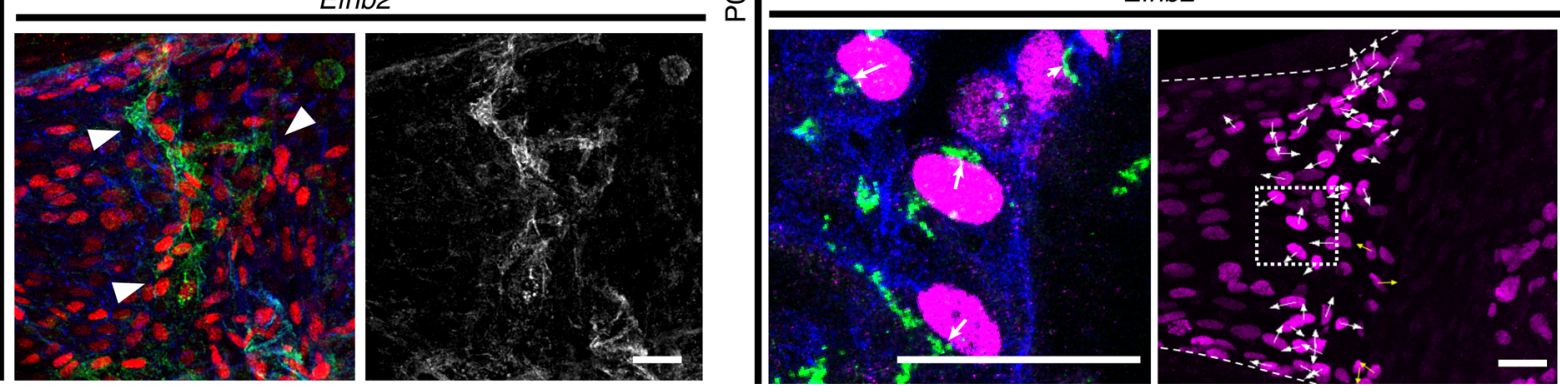

Figure 6. Failure of VFCs to project into vessel lumen and abnormal integrin expression. (A) Semithin longitudinal sections of PO femoral veins showed protruding VFCs in littermate controls, but no protruding cells were seen after homozygous Efnb2 deletion. 3D reconstructions of semithin sections show protruding VFCs (arrowheads) in controls only. The schematic indicates the orientation of the semithin sections. (B) A significant reduction in the mean number of sections showing protruding cells was identified ( $\geq 60$ sections were analyzed per sample, ${ }^{*} P<0.05$ by 2 -tailed $t$ test, $n=3 \mathrm{VV}$ per group, data are shown as mean \pm SEM). (C) Integrin $\alpha 9$ was expressed in a ring around the organized VFCs in littermate controls (white arrowheads), but after homozygous Efnb2 deletion, the localization of integrin $\alpha 9$ expression was disrupted and chaotic $\left(P<0.05, \chi^{2}\right.$ test of the proportion of VVs showing normal vs. disrupted integrin $\alpha 9$ expression pattern, $n \geq 6$ VV per group). (D) VFC polarity (indicated by white arrows) was examined by costaining for Prox1 (magenta), PECAM1 (blue), and Golgi (green). Polarity was determined for individual VFCs using $0.5-\mu m$ sections, and a $Z$ projection of 2-4 confocal sections shown on the right (area enlarged outlined by dotted box,). In littermate controls, cells in the central region of the vein were aligned with the line of organized VFCs, whereas after homozygous Efnb2 deletion, cell alignment was chaotic. $P<0.05$, $\chi^{2}$ test of the proportion of VVs showing normal vs. chaotic VFC alignment, $n \geq 8$ VV per group. Yellow arrows indicate VFCs on the posterior vein wall. Scale bars in A, C, and D: $20 \mu \mathrm{m}$. C and D are oriented as shown in Figure 2A. VFCs, valve-forming cells; VVs, venous valves. 
A

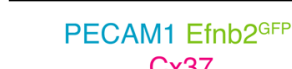
$\mathrm{C} \times 37$
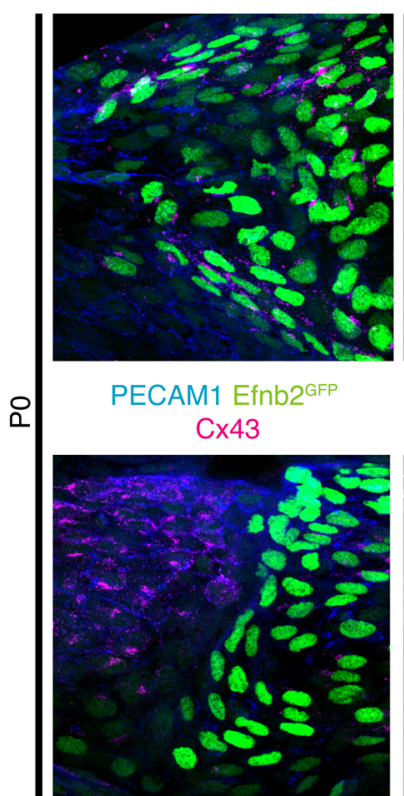

Control

C



Cx37

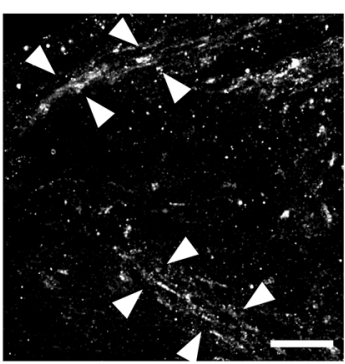

Cx43

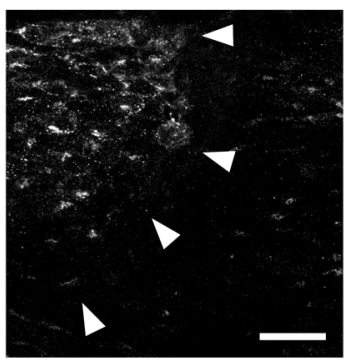

D
B

Prox1 PECAM1 Cx37

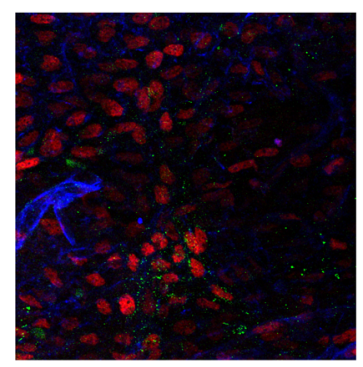

Prox1 PECAM1 Cx43

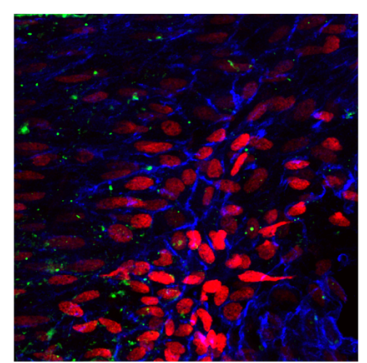

BLOOD FLOW

$\stackrel{\text { (all images) }}{\longrightarrow}$

Efnb2/x
Efnb2 $2^{|x| x}$

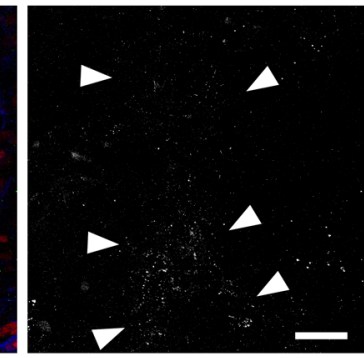

$\mathrm{C} \times 43$
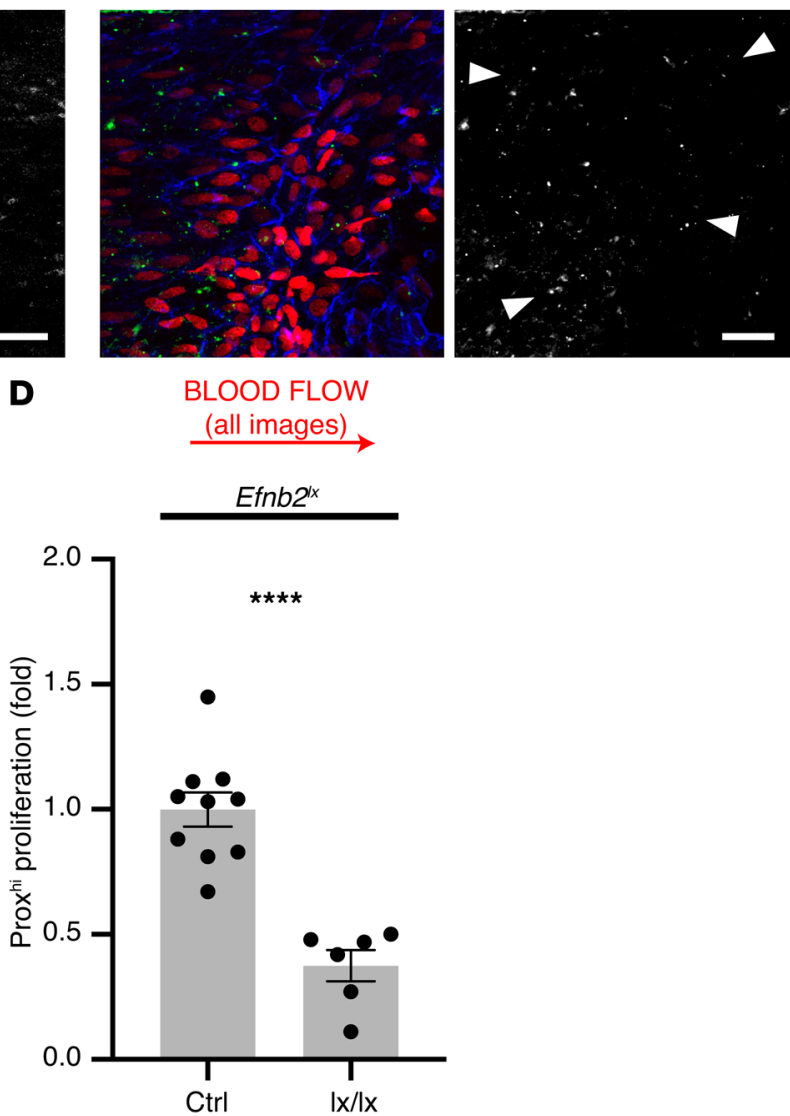

Figure 7. Efnb2 deletion disrupts gap junction protein expression pattern and proliferation. (A) Localization of PECAM1 (blue), and either Cx37 or Cx43, as indicated (magenta) around VFCs on PO in heterozygous Efnb2GFP (green) mice. As expected, on PO Cx37 was localized to Efnb2GFP-expressing VFCs, primarily forming large gap junction plaques (examples indicated between arrowheads) and Cx43 was localized to endothelium upstream of these VFCs (region to the left of the arrowheads). Smaller plaques are also identifiable. (B) Localization of PECAM1 (blue), Prox1 (red), and either Cx37 or Cx43, as indicated (green), after homozygous deletion of Efnb2. The tightly regulated expression pattern of $C \times 37$ was disrupted, with expression over a wider area (arrowheads) and the typical appearance of larger plaques was lost. The expression pattern of $[x 43$ was also disrupted and no longer confined to upstream of VFCs (arrowheads; $P<$ 0.05 , $\chi^{2}$ test of the proportion of VVs showing normal [confined] vs. disrupted expression pattern, $n \geq 6$ VV per group). (C and $\left.\mathbf{D}\right)$ The proportion of proliferating VFCs was assessed by colocalization of Prox1 and Ki67 (arrowheads). Ki67 $7^{+}$FCs were easily identified in littermate controls, but far fewer proliferating VFCs were identifiable after homozygous Efnb2 deletion. The inferior region of the vein is shown; ${ }^{* * *} P<0.00005$, unpaired 2 -tailed $t$ test, $n \geq 6$ VV per group, data are shown as mean \pm SEM). Scale bars in A-C: $20 \mu \mathrm{m}$. VFCs, valve-forming cells; VVs, venous valves; P0, postnatal day 0 .

of our study. In WT littermates VFC polarity was aligned with the boundary and developing ring of VFCs, whereas after Efnb2 deletion, VFC polarity was disorganized and cells were spread over a wider upstreamdownstream region. These results are consistent with previous in vitro findings, showing that the ephrinB2EphB4 interaction leads to separation and clustering of initially mixed populations of EphB4-expressing and 
ephrinB2-expressing ECs (47). In vitro, treatment with ephrinB2-Fc stimulates migration of HUVECS, and it is possible that ephrinB2 promotes the migration of VFCs (60).

It remains unknown how Efnb2 expression within veins is regulated. We have shown that the Efnb2 boundary forms as the VFCs organize, and it may be regulated by the VFCs themselves as they organize. Notably, BMP9 controls lymphatic remodeling and LV formation, and induces Efnb2 expression in lymphatic and blood endothelia in vitro, but it is not known whether there is a VV phenotype in $B m p 9^{-/-}$mice $(61,62)$. The extent to which there is proliferation of VFCs between E18 and P0, or whether there is de novo differentiation of new Prox $1^{\text {hi }}$ cells from surrounding endothelium, remains unclear.

Normal blood flow is required for postnatal VV maturation (11), and Efnb2-dependent protrusion of cells into the lumen on P0 could expose VFCs to higher fluid shear forces, particularly as the vessel lumen becomes more acutely narrowed (e.g., at stage 2 of VV development; ref. 12). Shear-regulated signaling might coordinate subsequent events in VV formation, for example, commissure formation. In embryonic stem cell-derived ECs in vitro, Efnb2 is upregulated by shear stress, which may contribute to the stimulation of VV leaflet growth postnatally $(12,63)$. This notion is consistent with the role of the oscillatory shear stress/Gata2/Foxc2 axis in LV endothelial differentiation, and the potential role of wall shear stress gradients in demarcating the locations of valve formation upstream of tributaries (64-66). Deletion of the mechanosensory ion channel Piezo1 results in defective VVs on P3, again consistent with a role for fluid shear in patterning VV (in addition to LV) formation $(57,67)$.

Signaling downstream of ephrin-Eph interactions can, for example, inhibit gap junction formation at the boundary between 2 cell populations, likely by cell repulsion preventing stable contacts between cells (32). It seems likely that cell-cell repulsion between ephrinB2 ${ }^{\text {hi }}$ VFCs and EphB4 ${ }^{\text {hi }}$;ephrinB2 ${ }^{\text {lo }}$ upstream cells on E18 patterns the migration of VFCs. It is unknown whether gap/junction signaling is important in this process, but loss of either $\mathrm{Cx} 37$ or $\mathrm{Cx} 43$ in mice leads to a similar phenotype with failure of VFC organization (11). Homozygous Efnb2 deletion disrupted the normally highly restricted expression patterns of Cx37 and $\mathrm{Cx} 43$ on $\mathrm{P} 0$, suggesting that gap junctional communication is disrupted. Gap junction plaque size varies depending on how many channels are clustered in the plaque. It is possible that plaques were present but much smaller, although this would also be expected to reduce cell-cell communication (68). We were unfortunately unable to develop experiments to demonstrate gap junctional VFC cell-cell communication in vivo, or confirm how this may be disrupted after deletion of Ephb4 or Efnb2.

Mutations in EFNB1 cause craniofrontonasal syndrome, whereas mice heterozygous for Efnb1 display skull defects that are thought to be mediated by inhibition of normal gap junctional communication via $\mathrm{Cx} 43$ at ectopic ephrin-Eph boundaries. EphrinB1 directly interacts with $\mathrm{Cx} 43$ and regulates its cellular distribution, and disruption of gap junction plaques was seen in $E f n b 1^{+/-}$mice (46). Although deletion of Efnb2 resulted in loss of large Cx37 plaques in VFCs, any direct interaction between ephrinB2 and $\mathrm{Cx} 37$ remains to be determined. Although not directly demonstrated in our experiments, it is reasonable to assume that after Efnb2 deletion, as the Prox $1^{\text {hi }}$ VFCs are further apart and are physically separated, there will be less communication between these cells via gap junctions (e.g., incorporating $\mathrm{Cx} 37$ ). EphrinB2 organizes VFC positioning and therefore facilitates the formation of functional gap junctions between adjacent VFCs. It is plausible, therefore, that disruption of connexin expression patterning and gap junctional communication may be part of the mechanism that underlies the phenotype seen after Efnb2 deletion (46).

In WT mice on P0, Cx43 was expressed upstream of the developing VV and was not clearly expressed by the Prox $1^{\text {hi }}$ VFCs that express ephrinB2 (11). Cx43 is clearly expressed by cells that also express EphB4. With deletion of Efnb2, Cx43 expression appeared more dispersed throughout the femoral vein, suggesting ephrinB2 is required for the restriction of the Cx43 expression domain. In cardiomyocytes, EphB4 physically associates with $\mathrm{Cx} 43$, and $\mathrm{EphB}$ activation inhibited cardiomyocyte gap junctional electrical coupling (69). It is possible that in upstream endothelia, signaling through EphB4 could inhibit gap junction communication via $\mathrm{Cx} 43$.

It is unclear why the VV phenotype after Ephb4 deletion was slightly weaker than that in Efnb2 deleted mice. EphrinB2 is more promiscuous, binding to EphB4, EphB3, and EphB2, whereas EphB4 exclusively interacts with ephrinB2 $(38,70)$. Isolated knockout of either Ephb2 or Ephb3 does not induce any cardiovascular phenotype, but a third of double knockouts have severely defective angiogenesis that resembles much of the phenotype of Efnb2-/- mice (38). EphB3 expression has been reported in veins (whereas EphB2 is expressed in nonvascular mesenchymal cells), but we could not detect specific signals for EphB2 or 
EphB3 in veins by immunohistology (data not shown). EphrinB2 regulates cell morphology and motility independently of binding its receptors in vitro, which could partly explain the stronger phenotype seen with Efnb2 deletion (71). In sprouting angiogenesis, ephrinB2 is required for endocytosis and signaling of other important regulators of EC function including Vegfr2 and Vegfr3 (which are expressed in developing VVs), and could play similar roles in VFC organization $(12,39,40)$. The slight difference in the phenotypes after deletion of Efnb2 and Ephb4 could be caused by differences in their protein stability, which we were unable to investigate, in part because of the lack of specific antibodies raised against ephrinB2. We could not confirm reduced Ephb4 or Efnb2 mRNA levels after conditional gene deletion, due to our inability to specifically isolate VV cells, but this has been confirmed for Efnb2 deletion in lymphatic endothelium (72).

Detachment of VFCs from their underlying basement membrane has previously been identified in LV formation, during angiogenesis, and we now show it here in VV formation $(48,73)$. Due to detachment, cell-cell contacts are highly restricted, and this is likely to impact cell/cell signaling processes $(48,74)$. In vitro, soluble ephrinB2-Fc acts antiadhesively, and the high ephrinB2 expression in VFCs could promote their detachment from the underlying basement membrane to facilitate reorientation and organization (47).

We have previously analyzed VFC nuclear reorientation and elongation in wholemount confocal microscopy to characterize phenotypes on P0/stage 1 of VV development (11). Here, we show that VFCs not only protruded into the vessel lumen at this stage, but that this protrusion was abolished after homozygous Efnb2 deletion. We also identify that ingress of interstitial cells was already occurring at this early stage and confirm their persistence in P6 and adult murine VV, and in adult human VV. Their existence has previously been demonstrated in human, rat, and rabbit VV, in contrast to LV, which lack interstitial cells $(2,49,54)$. In lymphovenous valve development, mural cells are recruited into the valve leaflets during maturation, but the developmental origin of these cells in VVs is currently unknown (54). The identity, origin and functions of these cells in VVs will be the subject of future studies.

Our data showing that EphB4 was required for postnatal development is consistent with the phenotype resulting from Efnb2 deletion on $\mathrm{P} 2$ or P0 $(11,12)$. Almost all Prox $1^{\text {hi }}$ and Foxc2 ${ }^{\text {hi }}$ VFCs were absent on P6, in contrast to deletion of Ppp3r1 (CnB1), in which a clear ring of Prox1- and Foxc2-expressing cells remains (11). This is consistent with a requirement for EphB4 (and ephrinB2) to develop/maintain the phenotype of free-edge cells to P6, rather than just growth of VV leaflets (11). The failure to establish a local reduction in the density of SMCs around the VV on P6 after Ephb4 deletion on P0 is consistent with the endothelial VFC/SMC signaling that controls this reduction in SMC density around LVs (31, 75-77).

Conclusions. In addition to an increased risk of lymphatic-related fetal hydrops, we show that patients carrying heterozygous mutations in EPHB4 had very few VVs, with early onset deep venous reflux indicating that the observed venous insufficiency was due to VV aplasia. By studying mice, it was demonstrated that ephrinB2 and EphB4 patterned the organization of VFCs on P0 and was required for cellular reorientation, elongation, protrusion, and proliferation, adding to our understanding of the complex VV developmental program. Postnatal deletion of Ephb4 led to complete loss of the valve, which could explain the phenotype observed in the patients.

\section{Methods}

Human VV ultrasonography. The brachial, basilic, popliteal, and short saphenous veins underwent ultrasonographic evaluation in London (Phillips IU22 with L17-5 MHz/L9-3 MHz probes) and VV maximum leaflet measurements obtained offline (Xcelera Cath Lab software, Phillips). Reproducibility was determined previously (11). For each vein, the number of VVs and VV length were normalized to the mean value in the respective control veins from our existing control population and additional new controls, and the mean number of VVs per vein, per patient, was compared. Deep venous (popliteal) reflux duration was measured bilaterally after distal manual compression while standing, and the mean was taken, with reflux defined as $0.5 \mathrm{sec}-$ ond or longer and severe reflux as longer than 1 second (78-80). Because deep venous reflux is rare, popliteal venous reflux was not routinely measured in the entire control population, but was subsequently measured in additional controls (81). Genotyping was performed at St George's, University of London and in Bergen (21).

Mouse lines. WT analyses were carried out in BALB/C mice obtained from Charles River UK. Prox$1 C r e E R^{T 2}$ (12), Rosa26 $6^{m T m G}$ (82), Efnb2 ${ }^{l x}$ (83), and Efnb2 ${ }^{G F P}$ (84) mice have been previously described and were maintained on C57BL/6 backgrounds. Tamoxifen/4OH-tamoxifen (in peanut or sunflower oil, MilliporeSigma) was injected i.p. either $1 \mathrm{mg}$ on E15 for analysis on P0, or $50 \mu \mathrm{g}$ on P0 for analysis on P6 in order to induce Cre activity in Prox $1 C r e E R^{T 2}$ mice (12). To delay labor, $37.5 \mu \mathrm{g} / \mathrm{g}$.Ms weight progesterone 
was given i.p. on E15 and E18 and embryos were analyzed on "E19," equivalent to P0. We compared VV in Prox $1 C r e E R^{T 2+}$ with Prox $1 C r e E R^{T 2-}$ littermate controls in all deletion experiments.

Electron microscopy. Mice were culled and perfused via the aorta with heparinized PBS (hPBS, $25 \mathrm{mg} / \mathrm{L}$; MP Biomedicals) prior to fixation overnight in glutaraldehyde $(2.5 \% \mathrm{v} / \mathrm{v}$ in $0.1 \mathrm{M}$ cacodylate buffer, $\mathrm{pH}$ $\left.7.4,4^{\circ} \mathrm{C}\right)$ and postfixation in osmium tetroxide $\left(1 \% \mathrm{w} / \mathrm{v}\right.$ in $0.1 \mathrm{M}$ cacodylate, $\left.\mathrm{pH} 7.4,4^{\circ} \mathrm{C}\right)$ for 1.5 hours. All samples were dehydrated through graded ethanols, equilibrated with propylene oxide, infiltrated with epoxy resin (TAAB), and polymerized at $70^{\circ} \mathrm{C}$ for 24 hours. Semithin sections $(0.45 \mu \mathrm{m})$ were cut and stained with $1 \%$ Toluidine Blue. For analysis of protruding VFCs, more than 90 serial semithin sections were analyzed per sample (2-tailed unpaired $t$ test). For $3 \mathrm{D}$ reconstructions on $\mathrm{P} 0$, semithin sections $(0.45 \mu \mathrm{m})$ were photographed (Leitz DMRB microscope, Micropublisher 3.3RTV camera), aligned in NIH ImageJ and reconstructed using Amira (Thermo Fisher Scientific). Ultrathin sections (50-70 nm, Reichert-Jung ultramicrotome) were mounted and contrasted using uranyl acetate/lead citrate for examination (Hitachi H7600, 80kV, AMT digital camera; ref. 85). For quantification of interstitial cells on P6, the length of the leaflet was measured in NIH ImageJ, and the number of whole interstitial cell nuclei was counted. Human great saphenous veins (obtained during coronary artery bypass grafting) were opened prior to processing as per murine samples, with visualization of ultrathin sections using a Hitachi S-3500N microscope.

IHC. Mice were culled and perfusion fixed via the aorta and femoral vein by perfusion with hPBS followed by $4 \%$ formaldehyde and then further fixed for 24 hours. The external iliac and femoral veins were excised and embedded in wax, and 5 - $\mu$ m sections were incubated with primary antibody and washed prior to amplification using polymer horseradish peroxidase (Menarini) and signal detection using SG peroxidase substrate (Vector). Sections were photographed using a Micropublisher 3.3RTV camera mounted on a Leitz DMRB microscope with PL Fluotar $\times 20$ lens (Leica). For human Connexin IHC, see Supplemental Methods.

Wholemount immunostaining and analysis. Mice were culled and perfused with hPBS via the aorta prior to fixation in $4 \%$ paraformaldehyde followed by blocking in 3\% v/v donkey serum, $0.3 \%$ Triton X-100, and further dissection prior to incubation with primary antibodies, and washing prior to localization with fluorophore-conjugated secondary antibodies. Samples were finally dissected and mounted in Prolong Gold (Invitrogen). Valves were imaged using a Leica SP5 confocal microscope $(1024 \times 1024$ resolution, 8-bit) to produce $\mathrm{Z}$ projections (NIH ImageJ) of median filtered (Leica LASAF/ImageJ, except for connexin localization or fluorescence quantification) stacks. Lookup tables were linear. Control samples were incubated with either the appropriate nonimmune $\operatorname{IgG}$ and then secondary antibody or streptavidin-conjugated fluorophore alone (Supplemental Figure 2A).

For analysis of VFC organization, Prox $1^{\text {hi }}$ nuclear elongation (proportion with length/width ratio greater than or equal to 2) and reorientation (proportion with long axis greater than or equal to $40^{\circ}$ from the vessel center line, in nuclei with length/width ratio greater than or equal to 2) were quantified in $Z$ projections (NIH ImageJ) as previously described $(11,48)$.

For analysis of Efnb2 $2^{G F P}$ expression on E18-P0 (Figure 4), $Z$ projections of confocal $Z$ stacks were oriented with flow left to right and the center line of the vessel horizontal. For each valve, $7-1210 \times 100-\mu \mathrm{m}$ regions of interest, each centered on the VFC upstream edge, were analyzed (NIH ImageJ). Mean intensity profiles for each fluorophore were converted to $Z$ scores and the mean of 6 VVs was plotted. On E18, areas with and without Prox $1^{\text {hi }}$ organizing VFCs were analyzed separately.

For analysis of areas of expression of Ephb4 and Efnb2-GFP on E18 (Figure 2B), an XZ projection (13.6- $\mu \mathrm{m}$ deep) across the reorientating VFCs was reconstructed (NIH ImageJ) and the relative fluorescence intensity profile for Efnb2-GFP and EphB4 was plotted. For quantification, for each valve 4-6 50- $\mu \mathrm{m}$ linear regions of interest were drawn, centered on the VFC leading edge, on E18, for $n=6$ VVs. Ephb4 upstream versus downstream intensity was compared (2-tailed $t$ test).

For analysis of cell orientation by coimmunostaining of nucleus and Golgi, stacks of $0.5-\mu m$ optical sections were analyzed (NIH ImageJ) to identify the Golgi for each VFC, and an arrow was drawn from nuclear center to Golgi center. The $Z$ projection of all arrows is shown.

Antibodies. The following antibodies were used: rabbit anti-Cx43 (Cell Signaling Technology 3512), Cx37 (CX37A11, Alpha Diagnostics), Prox1 (11-002P, Angiobio), ki67 (ab15580, Abcam), and Golgi apparatus protein 1 (ab103439, Abcam); sheep anti-Foxc2 (AF6989, R\&D); goat anti-EphB4 (BAF446, R\&D); rat anti-PECAM1 (clone MEC 13.3, BD); and mouse anti- $\alpha$ smooth muscle actin (clone 1A4 conjugated to Cy3, MilliporeSigma). For fluorescence signal detection, secondary antibodies or streptavidin were conjugated to Dylight-405/488/550/649 (Jackson ImmunoResearch). 
Statistics. For VV developmental stage 0-4 quantification, data represent the proportion of VV reaching each developmental stage. $P$ values represent the difference in proportion of valves at each stage versus their WT littermates ( $\chi^{2} /$ Fisher's exact test as appropriate). Comparisons of VFC nuclear elongation and reorientation between groups were analyzed by 1-way ANOVA and Bonferroni's post hoc tests. Age and sex matching for patient ultrasonography was tested respectively by 2-tailed unpaired $t$ test and Fisher's exact test. All analyses were carried out using IBM SPSS Statistics 24, and Graphpad PRISM v8. A $P$ value of less than 0.05 was considered significant. Ultrasonographers were blinded to participant genotype during scanning and image analysis/quantification. Experiments were not randomized.

Study approval. All human studies and animal studies were carried out in accordance with national regulations and ethical approvals in the United Kingdom and Sweden (Health Research Authority 12/LO/1164, 10/H0701/68, C130/15). Written informed consent was obtained from all participants.

\section{Author contributions}

OL, JW, CS, MI, AK, AA, MHV, MF, GVB, RAF, ASP, SP, PM, SJ, SB, SM, PO, TM, BM, PS, and AS designed research studies. OL, JW, CS, MI, AA, MHV, MF, AK, SP, and GVB conducted the experiments and acquired and analyzed data. PM, SJ, SB, SM, and PO provided the patients. OL, AS, and TM funded this study. OL, JW, CS, MI, AK, AA, MHV, MF, GVB, RAF, ASP, SP, PM, SJ, SB, SM, PO, TM, BM, PS, and AS reviewed drafts and approved the manuscript.

\section{Acknowledgments}

OL was funded by Academy of Medical Sciences Starter Grant for Clinical Lecturers (SGL016 1019), British Heart Foundation Centre of Research Excellence Travel Grant, and the Medical Research Council (G1000327).

Address correspondence to: Oliver Lyons, or Alberto Smith, Academic Department of Vascular Surgery, King's College London, 1st Floor North Wing, St Thomas' Hospital, London, SE1 7EH, United Kingdom. Phone: 44.0.2071880214; Email: oliver.lyons@cdhb.health.nz(OL); Email: alberto.smith@kcl.ac.uk (AS).

1. Phillips MN, et al. Micro-venous valves in the superficial veins of the human lower limb. Clin Anat. 2004;17(1):55-60.

2. Gottlob R, May R, eds. Venous Valves: Morphology, Function, Radiology, Surgery. Springer-Verlag Wien; 1986.

3. Eberhardt RT, Raffetto JD. Chronic venous insufficiency. Circulation. 2014;130(4):333-346.

4. Labropoulos $\mathrm{N}$, et al. The role of venous reflux and calf muscle pump function in nonthrombotic chronic venous insufficiency. Correlation with severity of signs and symptoms. Arch Surg. 1996;131(4):403-406

5. Friedman EI, et al. Congenital venous valvular aplasia of the lower extremities. Surgery. 1988;103(1):24-26.

6. Gorenstein A, et al. Congenital aplasia of the deep veins of lower extremities in children: the role of ascending functional phlebography. Surgery. 1986;99(4):414-420.

7. Bollinger A, et al. [Valve agenesis and dysplasia of leg veins. Morphological and functional studies]. Schweizerische medizinische Wochenschrift. 1971;101(37):1348-1353.

8. Plate G, et al. Congenital vein valve aplasia. World J Surg. 1986;10(6):929-934.

9. Meissner M, et al. The hemodynamics and diagnosis of venous disease. J Vasc Surg. 2007;46(6):S4-S24.

10. Lyons OT, et al. Redox dysregulation in the pathogenesis of chronic venous ulceration. Free Radic Biol Med. 2020;149:23-29.

11. Lyons O, et al. Human venous valve disease caused by mutations in FOXC2 and GJC2. J Exp Med. 2017;214(8):2437-2452.

12. Bazigou E, et al. Genes regulating lymphangiogenesis control venous valve formation and maintenance in mice. J Clin Invest. 2011;121(8):2984-2992.

13. Munger SJ, et al. Absence of venous valves in mice lacking Connexin37. Dev Biol. 2013;373(2):338-348.

14. Munger SJ, et al. Segregated Foxc2, NFATc1 and Connexin expression at normal developing venous valves, and Connexin-specific differences in the valve phenotypes of Cx37, Cx43, and Cx47 knockout mice. Dev Biol. 2016;412(2):173-190.

15. Goel RR, et al. Surgery for deep venous incompetence. Cochrane Database Syst Rev. 2015;2015(2):CD001097.

16. Geng X, et al. Intraluminal valves: development, function and disease. Dis Model Mech. 2017;10(11):1273-1287.

17. Connell FC, et al. The classification and diagnostic algorithm for primary lymphatic dysplasia: an update from 2010 to include molecular findings. Clin Genet. 2013;84(4):303-314.

18. Rosbotham JL, et al. Distichiasis-lymphoedema: clinical features, venous function and lymphoscintigraphy. Br J Dermatol. 2000;142(1):148-152.

19. Mellor RH, et al. Mutations in FOXC2 are strongly associated with primary valve failure in veins of the lower limb. Circulation. 2007;115(14):1912-1920.

20. Ostergaard P, et al. Rapid identification of mutations in GJC2 in primary lymphoedema using whole exome sequencing combined with linkage analysis with delineation of the phenotype. J Med Genet. 2011;48(4):251-255.

21. Martin-Almedina S, et al. EPHB4 kinase-inactivating mutations cause autosomal dominant lymphatic-related hydrops fetalis J Clin Invest. 2016;126(8):3080-3088. 
22. Amyere M, et al. Germline loss-of-function mutations in EPHB4 cause a second form of capillary malformation-arteriovenous malformation (CM-AVM2) deregulating RAS-MAPK signaling. Circulation. 2017;136(11):1037-1048.

23. Wooderchak-Donahue WL, et al. Phenotype of CM-AVM2 caused by variants in EPHB4: how much overlap with hereditary hemorrhagic telangiectasia (HHT)? Genet Med. 2019;21(9):2007-2014.

24. Yu J, et al. EPHB4 Mutation implicated in capillary malformation-arteriovenous malformation syndrome: a case report. Pediatr Dermatol. 2017;34(5):e227-e230.

25. Li D, et al. Pathogenic variant in EPHB4 results in central conducting lymphatic anomaly. Hum Mol Genet. 2018;27(18):3233-3245

26. Vivanti A, et al. Loss of function mutations in EPHB4 are responsible for vein of Galen aneurysmal malformation. Brain. 2018;141(4):979-988.

27. Duran D, et al. Mutations in chromatin modifier and ephrin signaling genes in vein of Galen malformation. Neuron. 2019;101(3):429-443.

28. Martin-Almedina S, et al. Janus-faced EPHB4-associated disorders: novel pathogenic variants and unreported intrafamilial overlapping phenotypes. Genet Med. 2021;23(7):1315-1324.

29. Luxan G, et al. Endothelial EphB4 maintains vascular integrity and transport function in adult heart. Elife. 2019;8:e45863.

30. Hirai H, et al. A novel putative tyrosine kinase receptor encoded by the eph gene. Science. 1987;238(4834):1717-1720.

31. Kania A, Klein R. Mechanisms of ephrin-Eph signalling in development, physiology and disease. Nat Rev Mol Cell Biol. 2016;17(4):240-256

32. Mellitzer G, et al. Eph receptors and ephrins restrict cell intermingling and communication. Nature. 1999;400(6739):77-81.

33. Xu Q, et al. In vivo cell sorting in complementary segmental domains mediated by Eph receptors and ephrins. Nature. 1999;399(6733):267-271.

34. Batlle E, Wilkinson DG. Molecular mechanisms of cell segregation and boundary formation in development and tumorigenesis Cold Spring Harb Perspect Biol. 2012;4(1):a008227.

35. Gerety SS, et al. Symmetrical mutant phenotypes of the receptor EphB4 and its specific transmembrane ligand ephrin-B2 in cardiovascular development. Mol Cell. 1999;4(3):403-414.

36. Gale NW, et al. Ephrin-B2 selectively marks arterial vessels and neovascularization sites in the adult, with expression in both endothelial and smooth-muscle cells. Dev Biol. 2001;230(2):151-160.

37. Shin D, et al. Expression of ephrinB2 identifies a stable genetic difference between arterial and venous vascular smooth muscle as well as endothelial cells, and marks subsets of microvessels at sites of adult neovascularization. Dev Biol. 2001;230(2):139-150.

38. Adams RH, et al. Roles of ephrinB ligands and EphB receptors in cardiovascular development: demarcation of arterial/venous domains, vascular morphogenesis, and sprouting angiogenesis. Genes Dev. 1999;13(3):295-306.

39. Wang Y, et al. Ephrin-B2 controls VEGF-induced angiogenesis and lymphangiogenesis. Nature. 2010;465(7297):483-486.

40. Sawamiphak S, et al. Ephrin-B2 regulates VEGFR2 function in developmental and tumour angiogenesis. Nature. 2010;465(7297):487-491.

41. Oike Y, et al. Regulation of vasculogenesis and angiogenesis by EphB/ephrin-B2 signaling between endothelial cells and surrounding mesenchymal cells. Blood. 2002;100(4):1326-1333.

42. Makinen T. PDZ interaction site in ephrinB2 is required for the remodeling of lymphatic vasculature. Genes Dev. 2005;19(3):397-410

43. Zhang G, et al. EphB4 forward signalling regulates lymphatic valve development. Nat Commun. 2015;6:6625.

44. Katsuta H, et al. EphrinB2-EphB4 signals regulate formation and maintenance of funnel-shaped valves in corneal lymphatic capillaries. Invest Ophthalmol Vis Sci. 2013;54(6):4102-4108.

45. Cowan CA, et al. Ephrin-B2 reverse signaling is required for axon pathfinding and cardiac valve formation but not early vascular development. Dev Biol. 2004;271(2):263-271.

46. Davy A, et al. Inhibition of gap junction communication at ectopic Eph/ephrin boundaries underlies craniofrontonasal syndrome. PLoS Biol. 2006;4(10):e315.

47. Fuller T, et al. Forward EphB4 signaling in endothelial cells controls cellular repulsion and segregation from ephrinB2 positive cells. J Cell Sci. 2003;116(pt 12):2461-2470.

48. Tatin F, et al. Planar cell polarity protein Celsr1 regulates endothelial adherens junctions and directed cell rearrangements during valve morphogenesis. Dev Cell. 2013;26(1):31-44.

49. Bazigou E, et al. Integrin-alpha9 is required for fibronectin matrix assembly during lymphatic valve morphogenesis. Dev Cell. 2009;17(2):175-186.

50. Bazigou E, et al. Primary and secondary lymphatic valve development: molecular, functional and mechanical insights. Microvasc Res. 2014;96:38-45

51. Francis R, et al. Connexin 43 modulates cell polarity and directional cell migration by regulating microtubule dynamics. $P L o S$ One. 2011;6(10):e26379.

52. Kanady JD, et al. Connexin37 and Connexin43 deficiencies in mice disrupt lymphatic valve development and result in lymphatic disorders including lymphedema and chylothorax. Dev Biol. 2011;354(2):253-266

53. Kanady JD, et al. Combining Foxc2 and Connexin37 deletions in mice leads to severe defects in lymphatic vascular growth and remodeling. Dev Biol. 2015;405(1):33-46.

54. Geng X, et al. Multiple mouse models of primary lymphedema exhibit distinct defects in lymphovenous valve development. Dev Biol. 2016;409(1):218-233.

55. Leu HJ. Familiar congenital absence of valves in the deep leg veins. Humangenetik. 1974;22(4):347-349

56. Fehnel KP, et al. Dysregulation of the EphrinB2-EphB4 ratio in pediatric cerebral arteriovenous malformations is associated with endothelial cell dysfunction in vitro and functions as a novel noninvasive biomarker in patients. Exp Mol Med. 2020;52(4):658-671

57. Nonomura K, et al. Mechanically activated ion channel PIEZO1 is required for lymphatic valve formation. Proc Natl Acad Sci U S A. 2018;115(50):12817-12822

58. Shadrina AS, et al. Varicose veins of lower extremities: Insights from the first large-scale genetic study. PLoS Genet. 2019;15(4):e1008110. 
59. Jones GT, et al. A variant of the castor zinc finger 1 (CASZ1) gene is differentially associated with the clinical classification of chronic venous disease. Sci Rep. 2019;9(1):14011.

60. Zheng LC, et al. Ephrin-B2/Fc promotes proliferation and migration, and suppresses apoptosis in human umbilical vein endothelial cells. Oncotarget. 2017;8(25):41348-41363.

61. Kim JH, et al. BMP9 induces EphrinB2 expression in endothelial cells through an Alk1-BMPRII/ActRII-ID1/ID3-dependent pathway: implications for hereditary hemorrhagic telangiectasia type II. Angiogenesis. 2012;15(3):497-509.

62. Levet S, et al. Bone morphogenetic protein 9 (BMP9) controls lymphatic vessel maturation and valve formation. Blood. 2013;122(4):598-607.

63. Masumura T, et al. Shear stress increases expression of the arterial endothelial marker ephrinB2 in murine ES cells via the VEGF-Notch signaling pathways. Arterioscler Thromb Vasc Biol. 2009;29(12):2125-2131.

64. Mahamud MR, et al. GATA2 controls lymphatic endothelial cell junctional integrity and lymphovenous valve morphogenesis through miR-126. Development. 2019;146(21):dev184218.

65. Sabine A, et al. Mechanotransduction, PROX1, and FOXC2 cooperate to control connexin37 and calcineurin during lymphatic-valve formation. Dev Cell. 2012;22(2):430-445.

66. Michalaki E, et al. Perpendicular alignment of lymphatic endothelial cells in response to spatial gradients in wall shear stress. Commun Biol. 2020;3(1):57.

67. Choi D, et al. Piezo1 incorporates mechanical force signals into the genetic program that governs lymphatic valve development and maintenance. JCI Insight. 2019;4(5):125068.

68. Solan JL, Lampe PD. Spatio-temporal regulation of connexin43 phosphorylation and gap junction dynamics. Biochim Biophys Acta Biomembr. 2018;1860(1):83-90.

69. Ishii M, et al. EphB signaling inhibits gap junctional intercellular communication and synchronized contraction in cultured cardiomyocytes. Basic Res Cardiol. 2011;106(6):1057-1068.

70. Sakano S, et al. Characterization of a ligand for receptor protein-tyrosine kinase HTK expressed in immature hematopoietic cells. Oncogene. 1996;13(4):813-822.

71. Bochenek ML, et al. Ephrin-B2 regulates endothelial cell morphology and motility independently of Eph-receptor binding. J Cell Sci. 2010;123(pt 8):1235-1246.

72. Frye M, et al. EphrinB2-EphB4 signalling provides Rho-mediated homeostatic control of lymphatic endothelial cell junction integrity. Elife. 2020;9:e57732.

73. Hirschberg RM, et al. Electron microscopy of cultured angiogenic endothelial cells. Microsc Res Tech. 2005;67(5):248-259.

74. Dong M, et al. Spatiomechanical modulation of EphB4-Ephrin-B2 signaling in neural stem cell differentiation. Biophys $J$. 2018;115(5):865-873.

75. Jurisic G, et al. An unexpected role of semaphorin3a-neuropilin-1 signaling in lymphatic vessel maturation and valve formation. Circ Res. 2012;111(4):426-436

76. Bouvree K, et al. Semaphorin3A, Neuropilin-1, and PlexinA1 are required for lymphatic valve formation. Circ Res. 2012;111(4):437-445

77. Petrova TV, et al. Defective valves and abnormal mural cell recruitment underlie lymphatic vascular failure in lymphedema distichiasis. Nat Med. 2004;10(9):974-981.

78. Sarin S, et al. Duplex ultrasonography for assessment of venous valvular function of the lower limb. Br J Surg. 1994;81(11):1591-1595.

79. Lurie F, et al. Multicenter assessment of venous reflux by duplex ultrasound. J Vasc Surg. 2012;55(2):437-445

80. van Bemmelen PS, et al. Quantitative segmental evaluation of venous valvular reflux with duplex ultrasound scanning. $J$ Vasc Surg. 1989;10(4):425-431.

81. Maurins U, et al. Distribution and prevalence of reflux in the superficial and deep venous system in the general population--results from the Bonn Vein Study, Germany. J Vasc Surg. 2008;48(3):680-687.

82. Muzumdar MD, et al. A global double-fluorescent Cre reporter mouse. Genesis. 2007;45(9):593-605.

83. Grunwald IC, et al. Hippocampal plasticity requires postsynaptic ephrinBs. Nat Neurosci. 2004;7(1):33-40.

84. Davy A, Soriano P. Ephrin-B2 forward signaling regulates somite patterning and neural crest cell development. Dev Biol. 2007;304(1):182-193.

85. Venable JH, Coggeshall R. A simplified lead citrate stain for use in electron microscopy. J Cell Biol. 1965;25:407-408. 